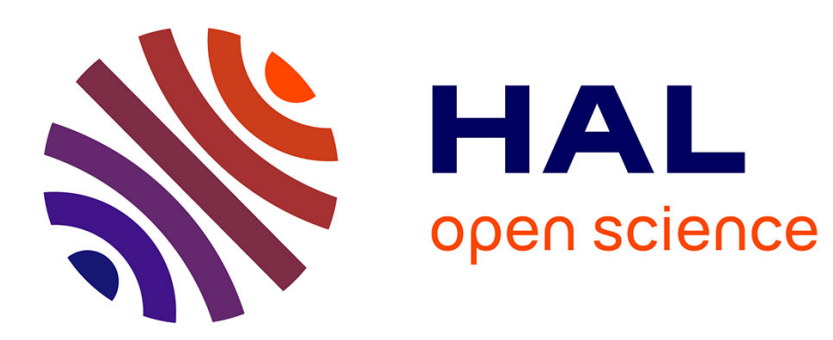

\title{
Characterizing most irregular small scale structures in turbulence using local Hölder exponents
}

Florian Nguyen, Jean-Philippe Laval, Bérengère Dubrulle

\section{To cite this version:}

Florian Nguyen, Jean-Philippe Laval, Bérengère Dubrulle. Characterizing most irregular small scale structures in turbulence using local Hölder exponents. Physical Review E , 2020, 102 (063105). hal$02514185 \mathrm{v} 2$

\section{HAL Id: hal-02514185 \\ https://hal.science/hal-02514185v2}

Submitted on 11 Dec 2020

HAL is a multi-disciplinary open access archive for the deposit and dissemination of scientific research documents, whether they are published or not. The documents may come from teaching and research institutions in France or abroad, or from public or private research centers.
L'archive ouverte pluridisciplinaire HAL, est destinée au dépôt et à la diffusion de documents scientifiques de niveau recherche, publiés ou non, émanant des établissements d'enseignement et de recherche français ou étrangers, des laboratoires publics ou privés. 


\title{
Characterizing most irregular small scale structures in turbulence using local Hölder exponents
}

\author{
F. Nguyen and J-P. Laval \\ Univ. Lille, CNRS, ONERA, Arts et Metiers Institute of Technology, Centrale Lille, \\ UMR 9014 - LMFL - Laboratoire de Mécanique des \\ fluides de Lille - Kampé de Fériet, F-59000 Lille, France \\ B. Dubrulle \\ SPEC, CEA, CNRS, Université Paris-Saclay, \\ CEA Saclay, 91190 Gif-sur-Yvette, France
}

\begin{abstract}
Two scalar fields characterizing respectively pseudo-Holder exponents and local energy transfers are used to capture the topology and the dynamics of the velocity fields in areas of lesser regularity. The present analysis is conducted using velocity fields from two Direct Numerical Simulations (DNS) of the Navier-Stokes equations in a triply periodic domain. A "typical irregular structure" is obtained by averaging over 213 most irregular events. Such structure is similar to a Burgers vortex, with non-axisymmetric corrections. A possible explanation of such asymmetry is provided by a detailed time-resolved analysis of birth and death of the irregular structures, which shows that they are connected to vortex interactions, possibly vortex reconnection.
\end{abstract}




\section{INTRODUCTION.}

Batchelor \& Townsend [1] speculated about the nature of small scale turbulent motions on the basis of hot wire velocity measurements in the Cavendish wind tunnel. Their main conclusion was that the energy associated with small scales is intermittent in space and time and organized into strong discrete vortices. Since then, progresses in computer power and image velocimetry has made it possible to investigate in more detail the nature and the properties of small scale turbulent motions, at scales of the order of or below the Kolmogorov scale. For example, it is now well established that regions where the vorticity supersedes the strain (the so-called Q criterion) are indeed organized into small scale elongated coherent structures that display a complex dynamics [2]. In some circumstances, they may interact and reconnect iteratively, following a self-similar vortex reconnection cascade. During reconnection, a distinct $-5 / 3$ inertial range is observed for the kinetic energy spectrum, associated with numerous resulting fine-scale bridgelets and thread filaments [3].

In the mean time, theoretical models of vortex reconnection using Biot-Savart model have evidenced a self-similar process, resulting in a near finite time singularity at the apex of the tent formed by the vortices [4]. Another evidence for quasi blow-up is provided by the "zeroth law of turbulence" [5], according to which the non-dimensional energy dissipation per unit mass becomes constant at large Reynolds number, implying a blow up of the enstrophy in the limit of zero viscosity. This suggests that the small scale structures of turbulent motions are very irregular, and connects to the question of the existence of singularities in the solutions to Navier-Stokes equations. Detecting and cataloging irregular structures found in numerical and experimental turbulence is therefore a first step in order to find a candidate for a mathematical non stationary singular solution to Navier-Stokes. This calls for specific tools to analyze the irregular structures. A suitable tool to deal with them was invented by Leray [6] and named "weak formulation". The main idea is to make a detour via the scale space, and work with a coarse version of the initial field (a "mollified" field), over a characteristic scale (resolution) $\ell$. At any given resolution $\ell$, the mollified field is sufficiently regular, so that all classical tools and manipulation of analysis of vector fields are valid. Limiting behaviors as resolution $\ell \rightarrow 0$ can then be used to infer results and properties for the rough field.

The problem of detection of turbulent irregular structures in numerical simulations has 
a long story. Most of these studies are conducted in the inviscid limit, using the Euler equations, where irregular structures are characterized by diverging vorticity [7-10]. A clear evidence for blow-up structure was claimed recently by [11] in an axisymmetric configuration, near a stagnation point. The relationship between diverging vorticity and vortex dynamics was investigated by Kerr (e.g. [12, 13]), while [14] uses an instanton method to capture the most probable "irregular structure". Fewer studies have been devoted to the possible topology of singularities or quasi-singularities in Navier-Stokes equations, characterized by diverging velocity. In particular, a study by [15] uses a criterion based on the geometry of the streamlines of velocity to search for singularities in Navier-Stokes turbulence. Another line of study is the analysis of the formation of extreme values of energy dissipation or vorticity with increasing Reynolds number [16]. More recently, Buaria et al. [17, 18] studied the formation of extreme events of velocity gradients in simulations of Navier-Stokes with a very good resolution. In the present paper, we build upon our previous work, where we showed how the mollified vector fields, over the scale $\ell$, can be used to follow extreme events of two scalar fields that encode the regularity properties of the small scale motions: i) a pseudo-Holder exponent $\tilde{h}(\mathbf{x})$ built using the Wavelet Transform Modulus Maxima (WTMM) method and providing the best local estimate of Hölder regularity compatible with the global multifractal analysis [see 19]; ii) a local energy transfer $D_{\ell}^{I}(\mathbf{x})$ built using the energy balance at scale $\ell$ of the weak solutions to Navier-Stokes equations [20, 21].

Here, we apply the tools based on local Hölder exponents to velocity fields issued from Direct Numerical Simulations (DNS) of the Navier-Stokes equations in a triply periodic domain, to capture the topology and the dynamics of the velocity fields in areas of lesser regularity. We further compute a "typical irregular structure" by averaging over 200 most irregular events. Such typical structure is similar to a Burgers vortex, with small nonaxisymmetric corrections. A possible explanation of such asymmetry is provided by a detailed time-resolved analysis of birth and death of the events, which shows that they are connected to vortex interactions, possibly vortex reconnections.

\section{TOOLS FOR STUDYING IRREGULAR MOTIONS}

In order to probe areas of lesser regularity in the flow, we use two different tools, based upon weak formulation and connected with the concept of Hölder continuity. A velocity 
field is said to be $h$-Hölder continuous with some exponent $h<1$ if there exists $C$ such that for $\ell$ small enough:

$$
\|\boldsymbol{u}(\mathbf{x}+\ell)-\boldsymbol{u}(\mathbf{x})\|<C \ell^{h}
$$

where $\boldsymbol{u}(\boldsymbol{x})$ is the velocity field and $\boldsymbol{\ell}$ is a vector of length $\ell$.

This regularity condition is intermediate between simple continuity and differentiability and is based on the velocity increment $\delta \mathbf{u}(\mathbf{x}, \boldsymbol{\ell})=\mathbf{u}(\mathbf{x}+\boldsymbol{\ell})-\mathbf{u}(\mathbf{x})$. Velocity fields with exponent $h<1$ are locally non-differentiable, but we can build derivatives in a weak sense by considering the quantity

$$
\nabla_{\ell} \boldsymbol{u}=-\int \nabla \Psi^{\ell}(\boldsymbol{y}) \boldsymbol{u}(\boldsymbol{x}, \boldsymbol{y}) d y
$$

where $\Psi^{\ell}(\boldsymbol{x})=\Psi(\boldsymbol{x} / \ell) / \ell^{3}$ and $\Psi$ is a regular, even, non negative function with norm 1 . In the sequel, we take $\Psi$ equal to a Gaussian for simplicity, so that we can make simple connection with classical wavelet transform. The weak derivative is well behaved for any $\ell>0$ and diverges locally as $\ell^{h-1}$.

This has various consequences in turbulent flows, that can be used to build useful local regularity indicators that we now discuss.

\section{A. The Duchon-Robert energy transfer.}

A first indicator of regularity properties of the velocity field is the local transfer of energy across scales. Indeed, a real singularity is expected to carry energy at a scale $\ell \rightarrow 0$. As a consequence, we expect that very irregular fields carry energy at scale below the Kolmogorov scale $\ell<\eta$ before it eventually gets dissipated through viscous effects.

This view was formalized by Duchon \& Robert [22]. They define local energy transfers from large to small scales at scale $\ell$ using a wavelet transform:

$$
D_{I}^{\ell}(\boldsymbol{x})=\frac{1}{4} \int \boldsymbol{\nabla} \Psi^{\ell}(\boldsymbol{y}) \cdot \delta \boldsymbol{u}(\boldsymbol{x}, \boldsymbol{y})\|\delta \boldsymbol{u}(\boldsymbol{x}, \boldsymbol{y})\|^{2} \mathrm{~d} \boldsymbol{y}
$$

It is worth stressing that this term should not be interpreted as the local transfer to a given scale $\ell$, analogous to the energy transfer function in [23]. Instead, it should be interpreted as the energy transfer from the field $\boldsymbol{u}_{\ell}(\boldsymbol{x})=\int \Psi^{\ell}(\boldsymbol{y}) \boldsymbol{u}(\boldsymbol{x}-\boldsymbol{y}) \mathrm{d} \boldsymbol{y}$ filtered at scale $\ell$ to the residual field $\boldsymbol{u}-\boldsymbol{u}_{\ell}$. 
Similarly, one can compute the energy locally dissipated by viscosity by the following [see 21]:

$$
D_{\nu}^{\ell}=\frac{\nu}{2} \int \nabla^{2} \Psi^{\ell}(\boldsymbol{y})\|\delta \boldsymbol{u}(\boldsymbol{x}, \boldsymbol{y})\|^{2} \mathrm{~d} \boldsymbol{y} .
$$

If the velocity is locally Hölder continuous with exponent $h, \delta u \sim \ell^{h}$, so then $D_{I}^{\ell} \sim \ell^{3 h-1}$ and $D_{\nu}^{\ell} \sim \nu \ell^{2 h-2}$. The two terms are balanced at a scale $\eta_{h} \propto \nu^{1 /(h+1)}$. The scale $\eta_{h}$ corresponds to the classical Kolmogorov scale $\eta$ for $h=1 / 3$, which is the value of $h$ predicted by Kolmogorov [24]. This also means that the scale $\eta_{h}$ gets lower than Kolmogorov scale if $h<1 / 3$. The physical meaning of $\eta_{h}$ is that of a "regularizing scale", below which the velocity increment transitions to a regular scaling dictated by a Taylor-expansion $\delta u \sim \ell \nabla u$. Note that a real singularity of the velocity field occurs with a Hölder coefficient of $h=-1$ since $\eta_{-1}=0$, so that the velocity field is never regularized.

In practice, a turbulent field follows Hölder continuity condition only for scales larger than $\eta_{h}$. Regions where the local energy transfer $D_{I}^{\ell}$ stays larger than the dissipation $D_{\nu}^{\ell}$ for a scale $\ell$ close to Kolmogorov scale are very irregular in the sense that their Hölder exponent is less than $h=1 / 3$. With such local indicator, we are therefore able to bound locally the regularity of the flow. However, we cannot derive a "hierarchy" of quasi-singular behavior, since we only have an upper bound on the Hölder exponent. To refine results, we need a more precise indicator.

\section{B. The local Hölder exponents.}

In a previous paper, we have developed a method to provide an estimate of the local Hölder exponent $\tilde{h}(\boldsymbol{x})$ using a local statistical method [19]. The scalar field computed using this method is continuous in space and shares the properties of the true Hölder exponent. In particular, the value of $\tilde{h}$ gets lower for areas of lesser local regularity, which makes it a good criterion to detect irregular events. We provide here only the general ideas that are necessary to understand how we estimate the local Hölder exponent and refer to [19] for technical details.

The physical idea is the following: if a velocity field is locally Hölder continuous with exponent $h$, its derivatives (in a weak sense) $\nabla_{\ell} u$ will locally blow-up and produce large deviations with respect to the most probable value (an "extreme event"). The smaller $h$ the faster the blow-up, so at a given small enough scale, we can expect that the intensity 
of the local weak derivative can be used to measure $h$. This argument is only qualitative, as it ignores constants that cannot be neglected when working at finite scale. To make it quantitative and calibrate the constants, we use multifractal theory.

For that, we introduce the scalar wavelet based velocity increment noted $\delta W_{\ell}(\boldsymbol{u})$ that is defined from the weak derivative as $\delta W_{\ell}(\boldsymbol{u})=\ell^{2}\left\|\nabla_{\ell}^{2} u\right\|$, corresponding to taking the norm of the Mexican hat wavelet transform of $u$. The probability of observing a large deviation for $\delta W_{\ell}(\boldsymbol{u})$ is then given by the rate function $C(h)$, obtained in the $\ell \rightarrow 0$ limit as

$$
\text { Prob }\left[\ln \left(\delta W_{\ell}(\boldsymbol{u})\right)=h \ln (\ell / L)\right] \sim e^{\ln (\ell / L) C(h)}=\left(\frac{\ell}{L}\right)^{C(h)} .
$$

In a turbulent flow, $C(h)$ is observed to have a parabolic shape, with a minimum $C(h)=0$ reached at the most probable Hölder exponent $h_{0} \equiv h(p=0)$, that is slightly shifted from the Kolmogorov value $1 / 3$ due to intermittency [see e.g. 25]. In the multifractal interpretation of Parisi \& Frisch [26], the quantity $D(h)=3-C(h)$ corresponds to the fractal dimension of the sub-space of Hölder exponent $h$. The multifractal spectrum is connected with the scaling exponents of the velocity structure function through a Legendre transform property: $\zeta(p)=\min _{h}(p h+C(h))$, where $\left\langle\left(\delta W_{\ell}(\boldsymbol{u})(\boldsymbol{x})\right)^{p}\right\rangle \sim \ell^{\zeta(p)}$.

The Legendre property can then be used to define the scale variation of a "typical amplitude" of the wavelet increments, connected with the most probable exponent $h_{0}$ as:

$$
\mathcal{T}_{0}(\ell)=\exp \left(\left\langle\ln \left(\delta W_{\ell}(\boldsymbol{u})\right)\right\rangle\right) \sim \ell^{h_{0}}
$$

More generally, one can define the scale variation of a "typical amplitude" of the wavelet increments, connected with the exponent $h(p)=d \zeta(p) / d p$ as:

$$
\mathcal{T}_{p}(\ell)=\exp \left(\frac{\left\langle\ln \left(\delta W_{\ell}(\boldsymbol{u})\right)\left(\delta W_{\ell}(\boldsymbol{u})\right)^{p}\right\rangle}{\left\langle\left(\delta W_{\ell}(\boldsymbol{u})\right)^{p}\right\rangle}\right) \sim \ell^{h(p)} .
$$

The higher the $p$, the deeper in the tail of the probability distribution function of $\delta W_{\ell}$ we dig to build $\mathcal{T}_{p}(\ell)$, so that we indeed probe the extreme deviations that we are looking for. Naively, we could therefore decide that if there exist a $p$ such that locally $\delta W_{\ell}=\mathcal{T}_{p}(\ell)$, then we can assign the local value $h=h(p)$ at such point. However, this is not so simple, because first the scaling is only defined up to a constant (you may multiply $\mathcal{T}_{p}(\ell)$ by any $c_{p}$ and get the same scaling, but a very different amplitude), and then the multifractal scaling is only valid in a statistical sense, so that there is no chance to match $\delta W_{\ell}$ and any $\mathcal{T}_{p}(\ell)$ over a range of scale. 
A coherent statistical construction then goes through the definition of active volumes $A_{p}$, characterizing regions where $\delta W_{\ell}$ exceeds a fraction of $\mathcal{T}_{p}(\ell)$ :

$$
\mathbf{x} \in A_{p} \quad \text { iff } \quad \delta W_{\ell}(\boldsymbol{u})(\boldsymbol{x}) \geq c_{p} \mathcal{T}_{p}(\ell)
$$

where $c_{p}$ is a scale independent constant, to be chosen later. Due to the scaling properties of $\mathcal{T}_{p}(\ell)$ (equation $(7)$ ), a point in a active volume $A_{p}$ has a local Hölder exponent less than $h(p)$. This leads us to interpret the boundaries of active volumes as sets of points with a given local Hölder exponent. The fractal dimension of those boundaries can further be measured using a box counting method. We then choose the coefficients $c_{p}$ such that the box counting dimensions from the active volumes $A_{p}$ matches the dimension extracted from multifractal spectrum $3-C(h(p))$.

The algorithm in the inertial range then proceeds in 4 s.pdf represented graphically in figure 1 for a given set of velocity fields:

- S1) Compute the associated multifractal spectrum $D(h)$ and the thresholds $\mathcal{T}_{p}(\ell)$ from the data.

- S2) Choose a value $\ell_{0}$ of $\ell$ in the inertial range and a set of values $\mathcal{T}$ spanning the interval $\left[0, \max \left(\delta W_{\ell_{0}}\right)\right]$; for each value of $\mathcal{T}$, draw the iso-countours $\delta W_{\ell_{0}}=\mathcal{T}$ and compute their associated box-counting dimension $D_{b c}(\mathcal{T})$.

- S3) For each $\mathcal{T}$, find $\tilde{h}_{\ell_{0}}$, so that $D_{b c}(\mathcal{T})=D\left(\tilde{h}_{\ell_{0}}\right)$ to build a function $\tilde{h}_{\ell_{0}}=f_{\ell_{0}}(\mathcal{T})$.

- S4) Associate a local exponent $\tilde{h}$ to any $\delta W_{\ell_{0}}$ via the formula $\tilde{h}_{\ell_{0}}=f_{\ell_{0}}\left(\delta W_{\ell_{0}}\right)$.

Note that while the local Hölder exponent $h$ cannot be continuous in the multifractal formalism, the exponent $\tilde{h}$ is continuous by definition. As a result, it is not a real measure of the Hölder exponent. However, it will still be relevant as a measure of the local regularity of the field, as discussed in [19].

\section{Generalization to low Reynolds number flow using extended self-similarity}

The method described in [19] is well adapted to high Reynolds number flows with a welldeveloped inertial range. However, it can be extended to lower Reynolds number flows, by using an empirical interesting universality property of the turbulent flows, called extended 
Calibration

(using the full dataset)

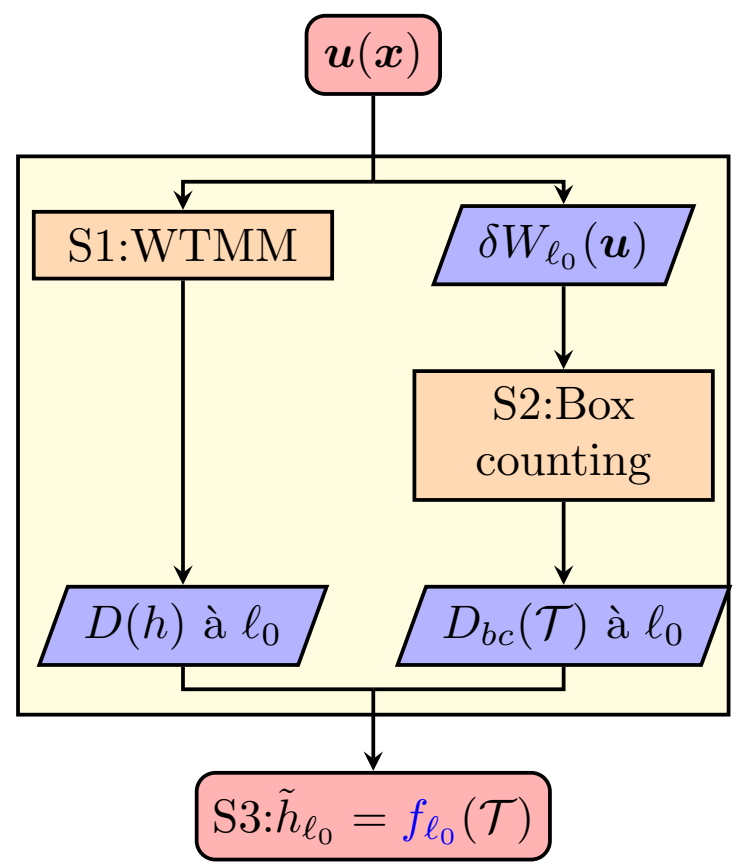

Application

(on any snapshot)

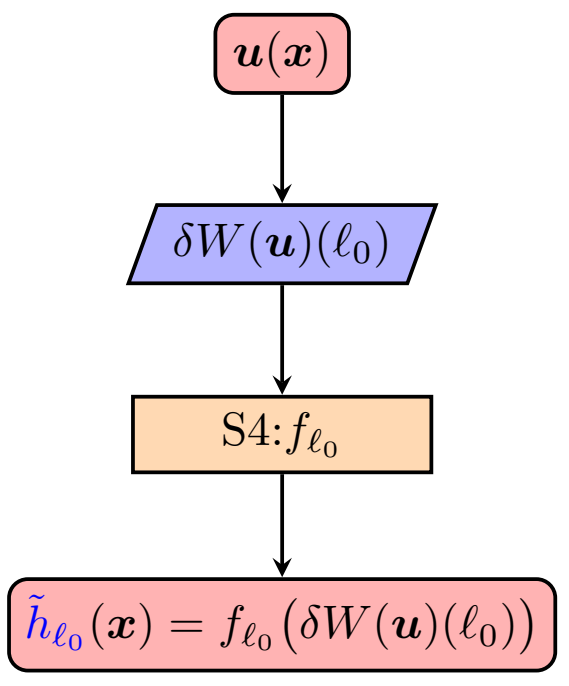

FIG. 1. Graphical representation of the algorithm for determining local Hölder exponent in the inertial range.

self-similarity [27], that is not fully understood yet. In the present case, it consists to realize that while the scaling $\mathcal{T}_{p}(\ell) \sim \ell^{h(p)}$ is valid only in the inertial range, it can be extended towards the dissipative range by considering the relative function $\mathcal{T}_{p}(\ell) / \mathcal{T}_{0}(\ell)$, that scales like $\ell^{h(p)-h_{0}}$. Due to the linearity properties of the active volume definitions, we can repeat all the s.pdf described in the previous section by considering the renormalized wavelet coefficient $\delta W_{\ell}(\boldsymbol{u})(\boldsymbol{x}) / \mathcal{T}_{0}(\ell)$ and thresholds $\mathcal{T}_{p}(\ell) / \mathcal{T}_{0}(\ell)$ and check that they belong to the same active volume than $\delta W_{\ell}(\boldsymbol{u})(\boldsymbol{x})$, while $C(h)$ is just translated by $h_{0}$. Through the procedure, we therefore get the relative exponent $\delta h=\tilde{h}-h_{0}$ for the quantity $\delta W_{\ell}(\boldsymbol{u})(\boldsymbol{x}) / \mathcal{T}_{0}(\ell)$, which enables to compute an effective scaling exponent $\tilde{h}=\delta h+\mathrm{d} \log \left(\mathcal{T}_{0}\right) / \mathrm{d} \log (\ell)$ at any scale $\ell$.

\section{Diving into the dissipative scales}

While the notion of Hölder exponent only makes sense in the inertial range of scales, it is useful to generalize it at dissipative scales, so as to probe regularity properties of the flow at such scales. To do so, we use the fact that $c_{p}$ is scale independent and the extended-self- 
similarity (ESS) to "follow down the scales" an irregular event down to the dissipative scale $\ell_{d}$. The algorithm in the dissipative range is then described by adding the following s.pdf:

- S5) Determine the regularity indicators $h_{\ell_{0}}(p)$ and $h_{\ell_{d}}(p)$ using the fit of power laws corresponding to $h(p)=\mathrm{d} \log \left(\mathcal{T}_{p}\right) / \mathrm{d} \log (\ell)$ or equivalently, using ESS, as $h(p)=$ $\mathrm{d} \log \left(\mathcal{T}_{p} / \mathcal{T}_{0}\right) / \mathrm{d} \log (\ell)+\mathrm{d} \log \left(\mathcal{T}_{0}\right) / \mathrm{d} \log (\ell)$.

- S6) In the inertial range, for each $p$, find $c_{p}$ by imposing that $c_{p} \mathcal{T}_{p}\left(\ell_{0}\right)=f_{\ell_{0}}^{-1}\left(h_{\ell_{0}}(p)\right)$.

- S7) Build the calibration function $f_{\ell_{d}}$ by plotting, for each $p, h_{\ell_{d}}(p)$ as a function of $c_{p} \mathcal{T}_{p}\left(\ell_{d}\right)$. The calibration function is then the smooth curve going through all the points such that $h_{\ell_{d}}(p)=f_{\ell_{d}}\left(c_{p} \mathcal{T}_{p}\left(\ell_{d}\right)\right)$. It can then be used to associate to each value of $\delta W_{\ell_{d}}$ a local Hölder exponent $\tilde{h}\left(\delta W_{\ell_{d}}\right)$ via the link $\tilde{h}\left(\delta W_{\ell_{d}}\right)=f_{\ell_{d}}\left(\delta W_{\ell_{d}}\right)$.

The complete algorithm is represented graphically in figure 2. The determination of the local Hölder exponent at the scale $\ell_{d}$ proceeds similarly to step S4 using this new function $f_{\ell_{d}}$. Because the flow regularizes as the scale decreases, the "local exponent" $\mathrm{d} \log \left(\mathcal{T}_{p}\right) / \mathrm{d} \log (\ell)$ will gradually change from $h_{\ell_{0}}(p)$ to 2 (such value can be measured thanks to the use of the Mexican wavelet transform and corresponds to a twice differentiable velocity field). Therefore, the flow will be considered very irregular at dissipative scale whenever the effective regularity indicator will be smaller than 2 .

\section{COMPUTATION OF THE LOCAL HÖLDER EXPONENTS}

In the sequel, we apply our method to the two simulations described by Nguyen et al. [19]. For the sake of convenience, the parameters for the simulation are reported in the table

I. The corresponding energy spectra are shown in figure 3. Run I has a larger Reynolds number, but a small dissipative range. Run II has a smaller Reynolds number, but an extended dissipative range. In the sequel, we use Run I to illustrate the outcome of our method in the inertial range, while we use Run II to dive into the dissipative range. Run II in particular is split in two parts. In the first part, we extracted 40 velocity fields at least 3 eddy turn over times $t_{\eta}=(\nu / . p d f \text { ilon })^{1 / 2}$ apart for the computation of all statistics. As another objective is to study the time evolution of potentially singular events, the simulation is extended in order to collect 100 snapshots of time resolved data (saved every $0.15 t_{\eta}$ ). 


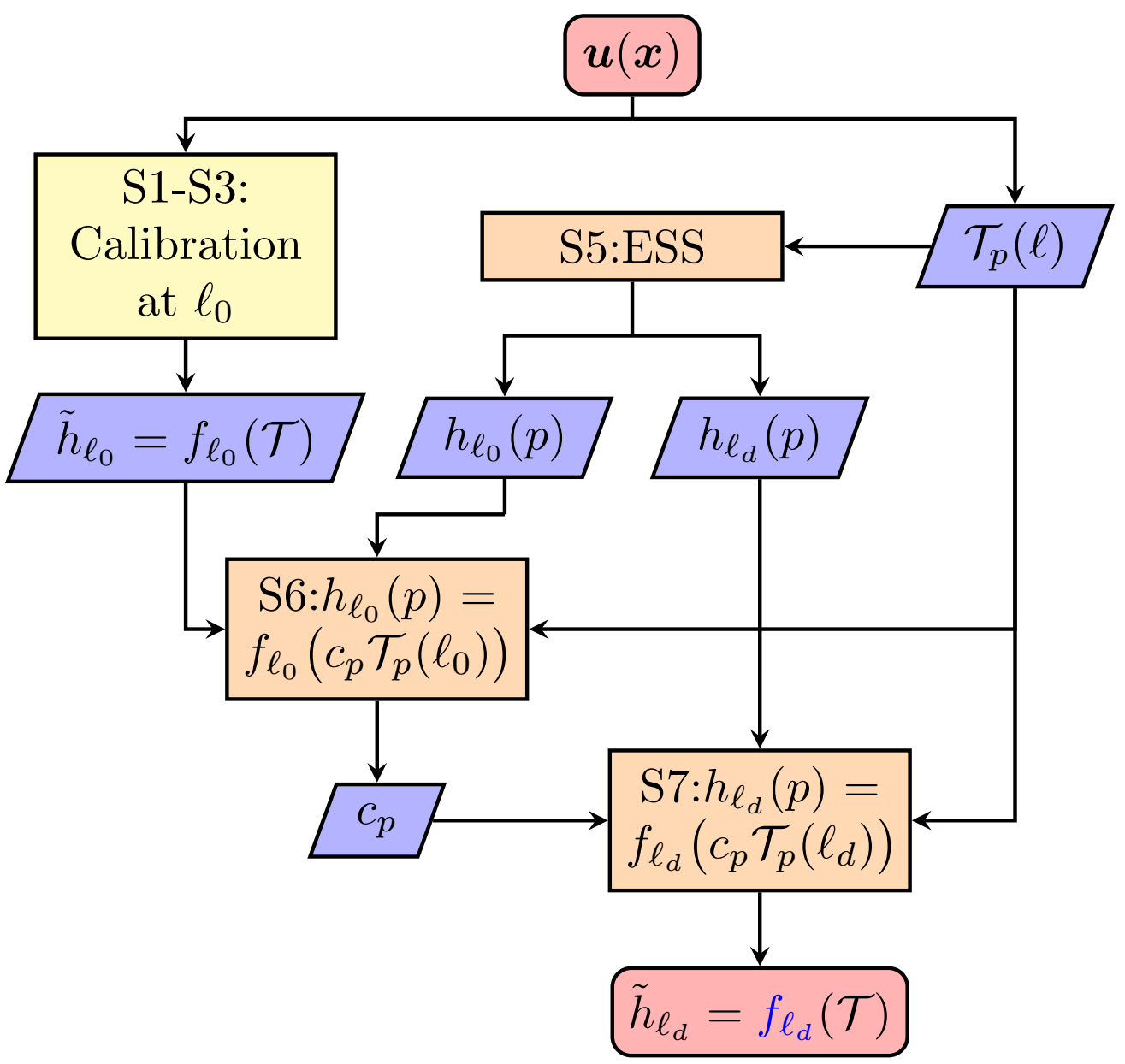

FIG. 2. Graphical representation of the algorithm for determining local Hölder exponent in the dissipative range.

\begin{tabular}{|c|c|c|c|c|c|c|c|c|}
\hline Run & $L$ & $\lambda$ & $\eta$ & $\left\langle u^{2}\right\rangle^{1 / 2}$ & .pdfilon & $\eta k_{\max }$ & $R e_{L}$ & $R e_{\lambda}$ \\
\hline I & 0.79 & 0.19 & 0.0083 & 0.54 & 0.089 & 2.1 & 570 & 140 \\
\hline II & 0.94 & 0.48 & 0.034 & 0.55 & 0.097 & 8.5 & 104 & 53 \\
\hline
\end{tabular}

TABLE I. Parameters used in the simulations with resolution $768^{3}$. With dealiasing, the cutoff wavenumber is $k_{\max }=256$. $L$ is the integral scale, $\lambda$ is the Taylor scale, $\eta$ is the Kolmogorov scale, $\left\langle u^{2}\right\rangle^{1 / 2}$ is the rms velocity, .pdfilon is the energy dissipation rate, $\eta k_{\text {max }}$ characterizes the resolution $\left(\eta k_{\max }>\pi\right.$ is resolved at Kolmogorov scale), $R e_{L}$ is the Reynolds number based on the integral scale, and $R e_{\lambda}$ is the Taylor based Reynolds number. 


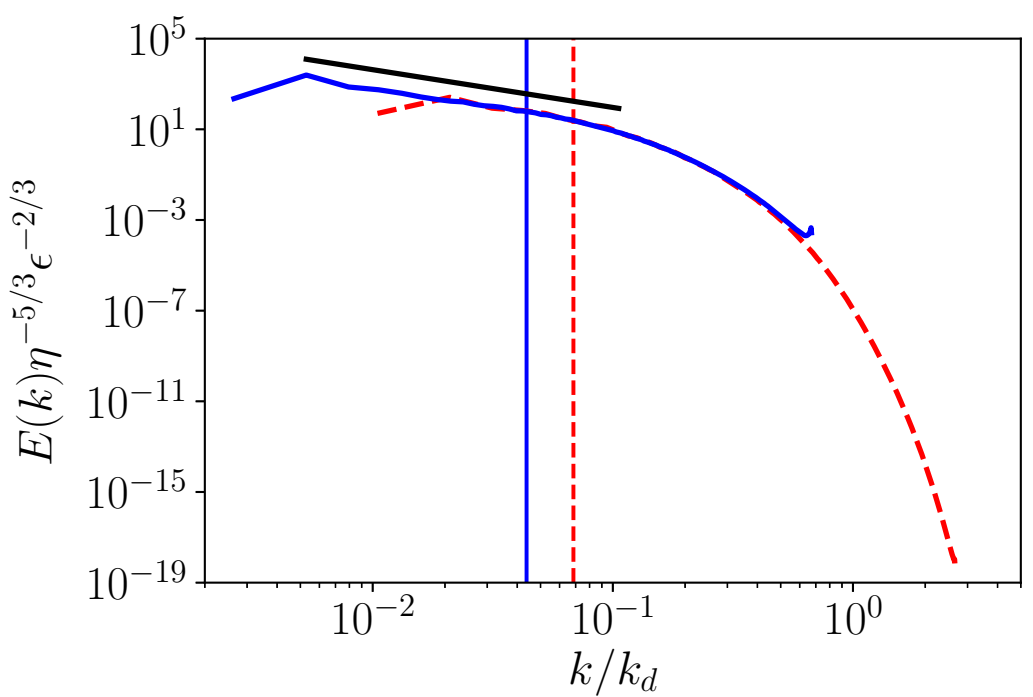

FIG. 3. Energy spectra for the two simulations. The blue continuous (resp. red dashed) curve stands for the simulation in the inertial (resp. dissipative) range. The black line materializes the $k^{-5 / 3}$ slope for the inertial range. The vertical lines materialize the Taylor scale wave number $k_{\lambda}$ for each simulation.

\section{A. Calibration of the local Hölder.}

In the sequel, all wavelets transforms are performed using a Mexican wavelet. The first three moments of the Mexican wavelet are null, which allows to measure Hölder exponents up to 3 [28]. The final outcome of step S1 to S3 for the two runs are summarized in figure 4, so as to have a clear synthetic view of the differences and similarities between the two runs.

The computation of the multifractal spectrum $(\mathrm{S} 1)$ is done using the WTMM method [25]. Because the inertial range is much shorter in Run II than in Run I, computations are performed at a scale closer to the injection scale. As such, the convergence of the WTMM method is hard to reach, as there are much fewer lines of maxima compared to the previous case. In order to reach the convergence, 40 velocity fields uncorrelated in time were used. We further verify that adding 60 more velocity fields from the time resolved dataset do not modify the results significantly. This does not prevent the effect of the proximity to the injection scale, which is emphasized by the fact that the most probable Hölder coefficient for Run II in figure 4 a drops to $h=0.3$. This value is significantly lower than for Run I and may affect the other s.pdf. As such, we need to keep in mind that the estimation of the local 
Hölder exponents may be inaccurate. The steps S2 and S3 are then implemented, leading to the calibration function for the inertial scale shown in figure 4c. The implementation of the step S5 is then performed using the extended self-similarity property, shown in figure 5a for Run I and figure 7a for Run II. In both cases, a power law, compatible with the prediction of section II C is obtained yielding the quantity $\delta h(p)=h(p)-h_{0}$. The reference value $h_{0}$ is then evaluated by fitting a power law over the thresholds $\mathcal{T}_{0}(\ell)$ at a chosen scale. Finally, the coefficient $c_{p}$ are computed using step S6, and illustrated on figure $4 \mathrm{~d}$. We see that their variation with $p$ seem to depend on the Reynolds number. More simulations at different Reynolds are needed to clarify this point, but this is out the scope of the present paper.

Using these results, we may then get a cartography of the local scaling exponent, by applying step S4. In figure 6, we provide here an illustration of an event detected in Run I corresponding to a low value of $h$ in the inertial range. This representation includes both the local Hölder exponents and local energy transfers introduced in section II. We see that it corresponds to a swirling regions organized around a filamentary region of low regularity, and characterized by a strong negative (inverse) local energy transfer at the center, surrounded by regions of high positive (direct) local energy transfer.

We now focus on the Run II, and use the procedure described in section II D to obtain an estimate of $\tilde{h}$ near the dissipative scale. Here, we adopt $\ell_{d}=1.8 \eta$, which is as close to the Kolmogorov scale as we can afford without losing accuracy on the Mexican wavelet used for computations. Applying S5 at this new scale, we then computed $h_{0}$ at this scale from a local power law fit of $\mathcal{T}_{0}(\ell)$ around such scale, and obtained $\tilde{h}$ by the appropriate subtraction. Finally, applying step S7, we get the calibration function $f_{\ell_{d}}$ shown in figure 8 . From the results and even if we take into account the uncertainty on the exact value of local Hölder exponent, it is clear that events with $\tilde{h}<2$ exist at this scale. Such events are interpreted as locations where the velocity field is not twice differentiable at this scale.

As the objective of this paper is to probe for very singular events at the dissipative scale, we want to give an estimation of the local Hölder exponent even if the rescaled velocity increment $\mathcal{T} /(\text {.pdfilon } \ell)^{1 / 3}$ is greater than 1.2. For this purpose, we choose to extrapolate the matching function up to $\tilde{h}=1.2$ (corresponding to a value $\tilde{h} \sim 0.1$ in the inertial range) as shown in figure 8. As such large increments are expected to be rare and very localized in space (see figure 6), we deem a simple linear extrapolation to be sufficient for our purpose. Note that we are unable to associate values of local Hölder exponents to very small velocity 


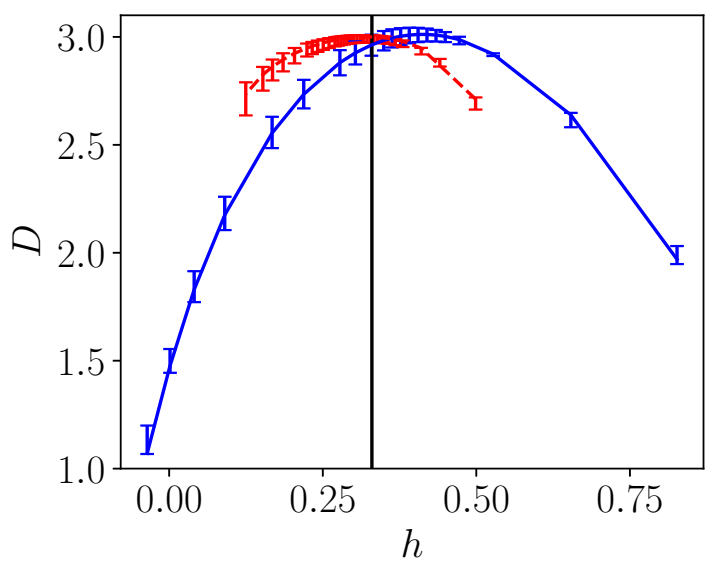

(a)

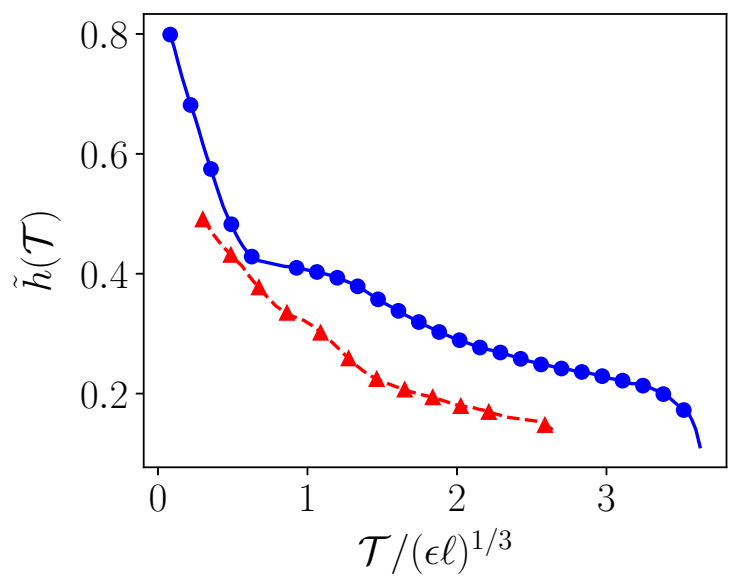

(c)

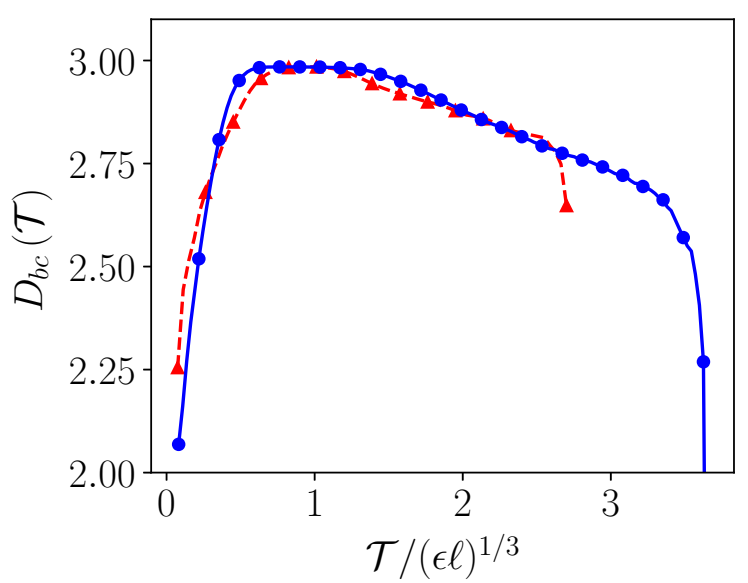

(b)

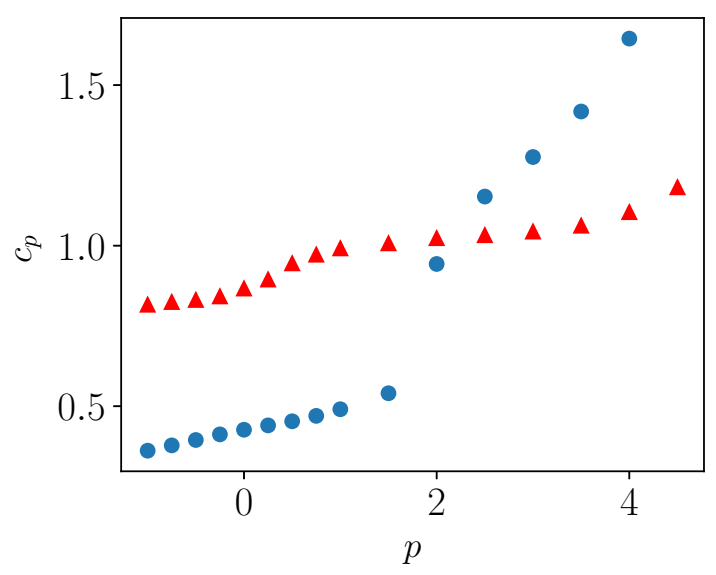

(d)

FIG. 4. Results related to Run I (resp. Run II) are shown in blue with continuous lines and circular markers (resp. red with dashed lines and triangular markers). (a) Multifractal spectrum fitted from $\ell \approx 1.2 \lambda$ to $\ell \approx 1.4 \lambda$. The vertical line materializes $h=1 / 3$. The error bars correspond to a shift of the fitting range by $5 \%$ for the power laws. (b) Box counting dimensions evaluated at the same scale. The velocity increments are normalized by the characteristic velocity at this scale. (c) Matching function resulting from the combination of (a) and (b). (d) Coefficients $c_{p}$ obtained by combining the results of (c) with the thresholds $\mathcal{T}_{p}(\ell)$. 


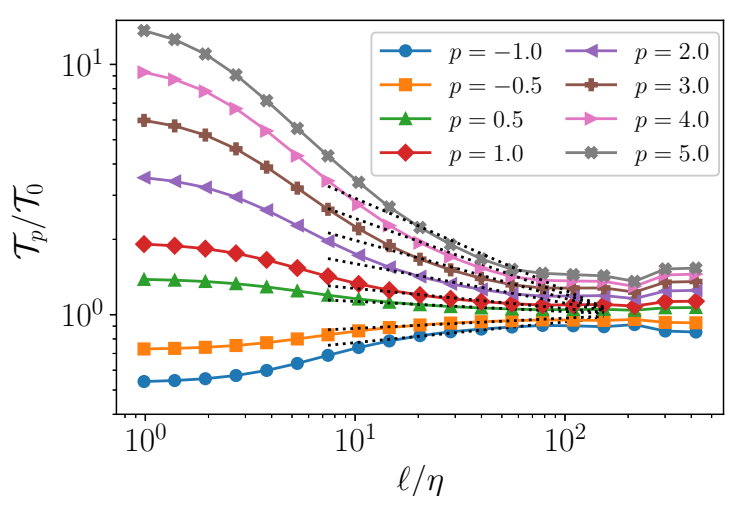

(a)

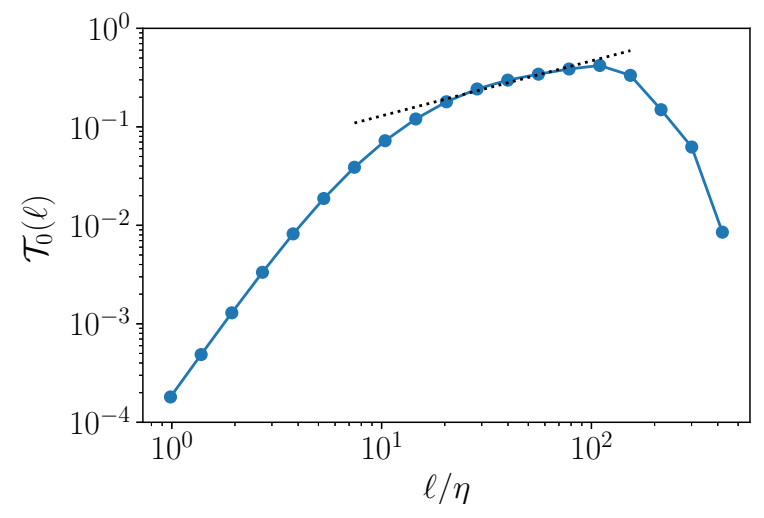

(b)

FIG. 5. (a) Values of the threshold $\mathcal{T}_{p}(\ell)$ as a function of scale for several $p$ computed over 10 snapshots of run I. The thresholds are renormalized by $\mathcal{T}_{0}(\ell)$. Fits are given in dotted lines. (b) Reference thresholds $\mathcal{T}_{0}(\ell)$. The fit used in the calibration of the method is materialized by a dotted line.

increments. This is because such velocity increments are also rare, corresponding to the $p<0$ branch of the multifractal spectrum. This lack of information about large values of local Hölder exponents will not affect our study of very irregular events.

In the sequel, we use the matching function shown in figure 8 to cartography and characterize areas of low regularity at the dissipative scale. For this, we use step S5 to get maps of $\tilde{h}$ at the dissipative scale. Detailed cartography of very irregular regions at the dissipative scale are provided in the supplementary material. They all appear to be associated with filament like vortices. Before discussing their individual properties and their possible origin, we first perform statistical analysis of the corresponding velocity fields.

\section{CLASSIFICATION USING THE VELOCITY GRADIENT TENSOR INVARI- ANTS}

As a first step, we may try to make a classification of the most irregular velocity fields by using the Velocity Gradient Tensor (VGT) invariants, as first suggested by Debue [29] in the case of experimental datasets, and using local energy transfer as regularity indicators. The VGT invariants, first described by Chong et al. [30], allow to classify the local topology of the streamlines of velocity in four categories: "vortex stretching", "vortex compressing", 


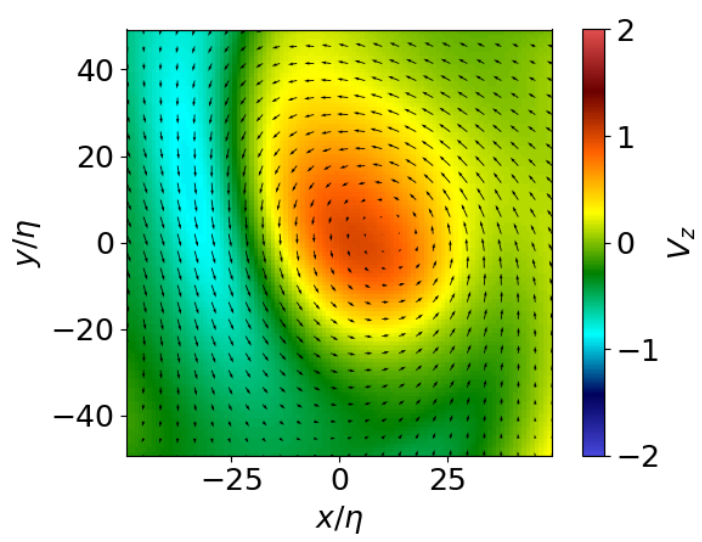

(a)

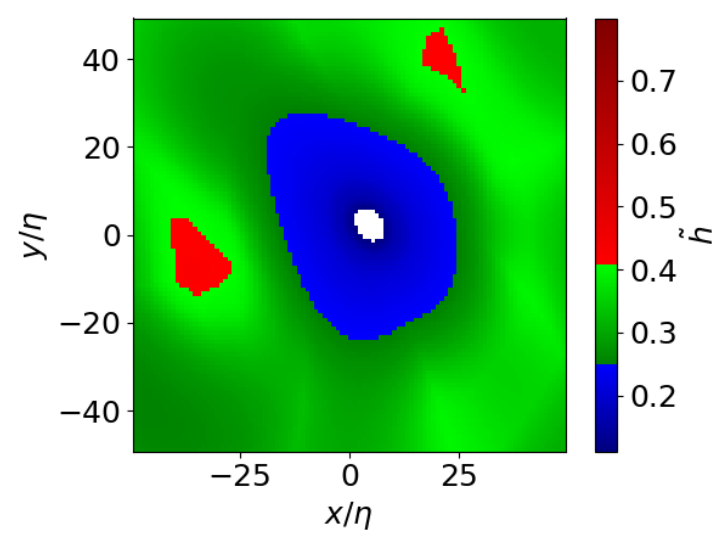

(c)

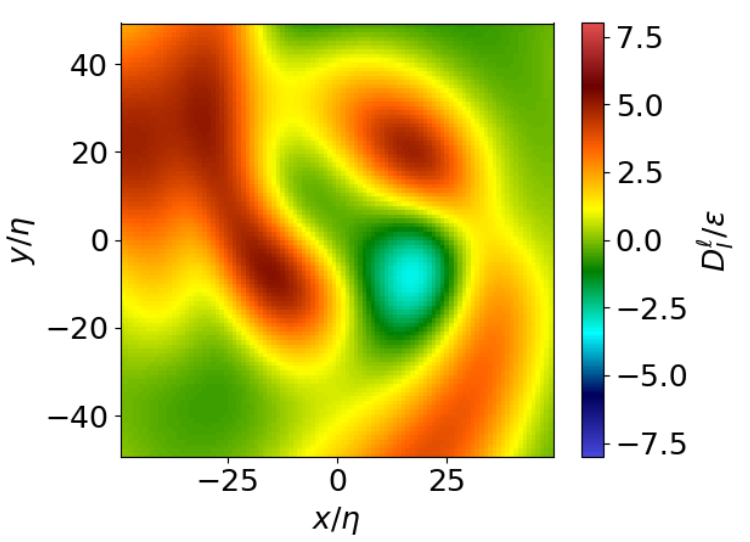

(b)

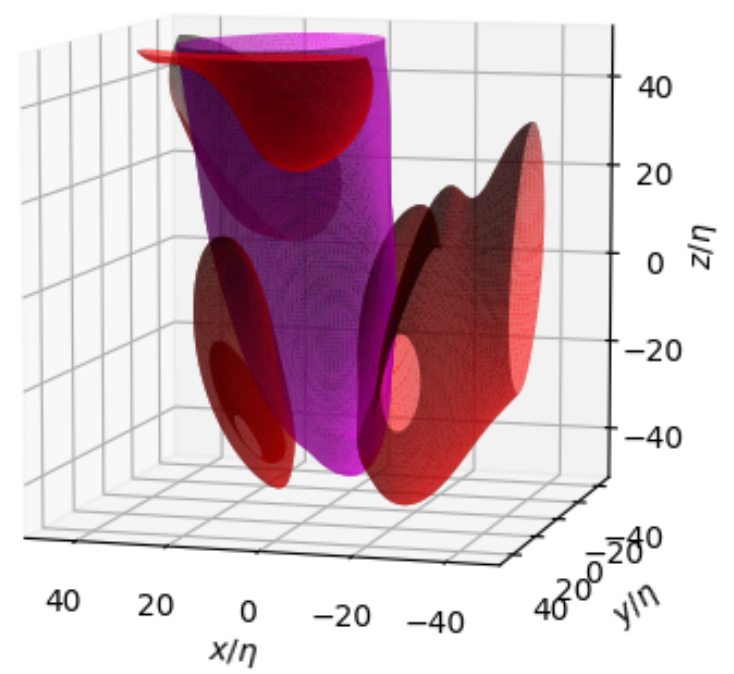

(d)

FIG. 6. Region with strong irregularity at $\ell=1.4 \lambda$ in the inertial range extracted from one snapshot of Run I. (a) Velocity along a slice passing through the point of maximal velocity increment (corresponding to an undetermined minimal value of local Hölder exponent). The arrows stand for the in-plane velocity and the colormap for the third component of velocity. (b) Local energy transfers on the same slice. (c) Local Hölder exponents on the same slice. White area corresponds to strong velocity increments which cannot be associated to a value of $\tilde{h}$ using the mapping function of figure 4c. (d) 3D representation of the irregular region. The magenta isosurface corresponds to $\tilde{h}=0.25$. The red isosurface corresponds to $D_{I}^{\ell}=4$.pdfilon. 


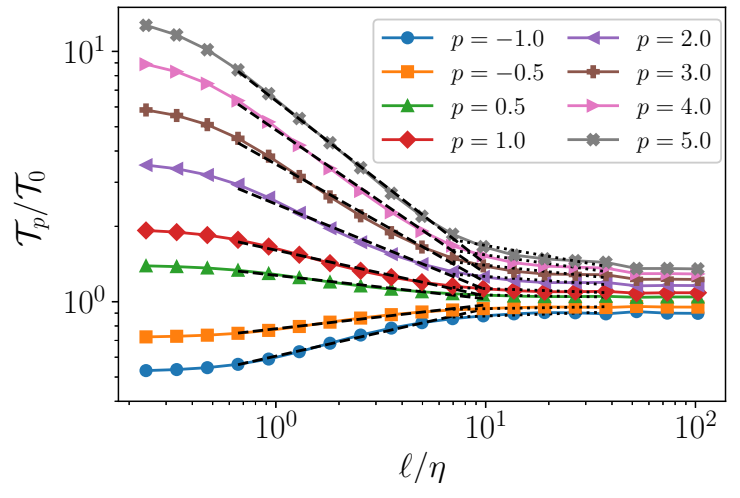

(a)

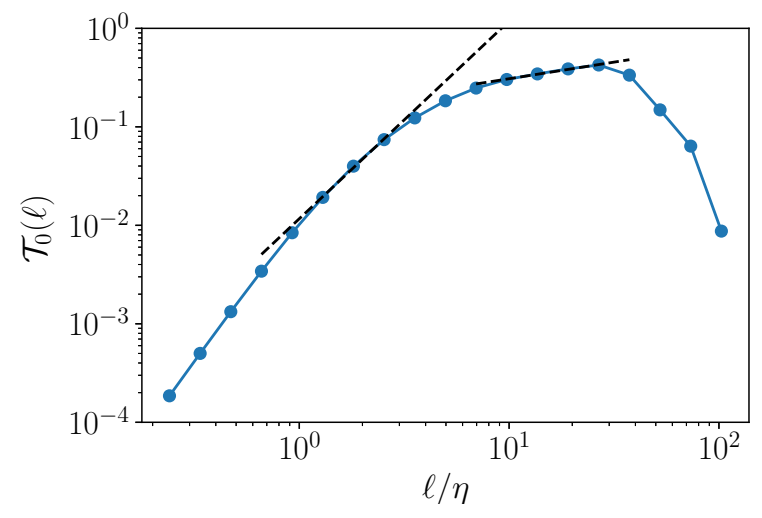

(b)

FIG. 7. (a) Values of the threshold $\mathcal{T}_{p}(\ell)$ for the global increments as a function of scale for several $p$ computed over Run II. The thresholds are renormalized by $\mathcal{T}_{0}(\ell)$. (b) Reference thresholds $\mathcal{T}_{0}(\ell)$. Fits corresponding to the inertial range, associated to $\ell_{0} \approx 1.4 \lambda$ are given in dotted lines while fit in the dissipative range associated to $\ell_{d}=1.8 \eta$ are given in dashed lines.

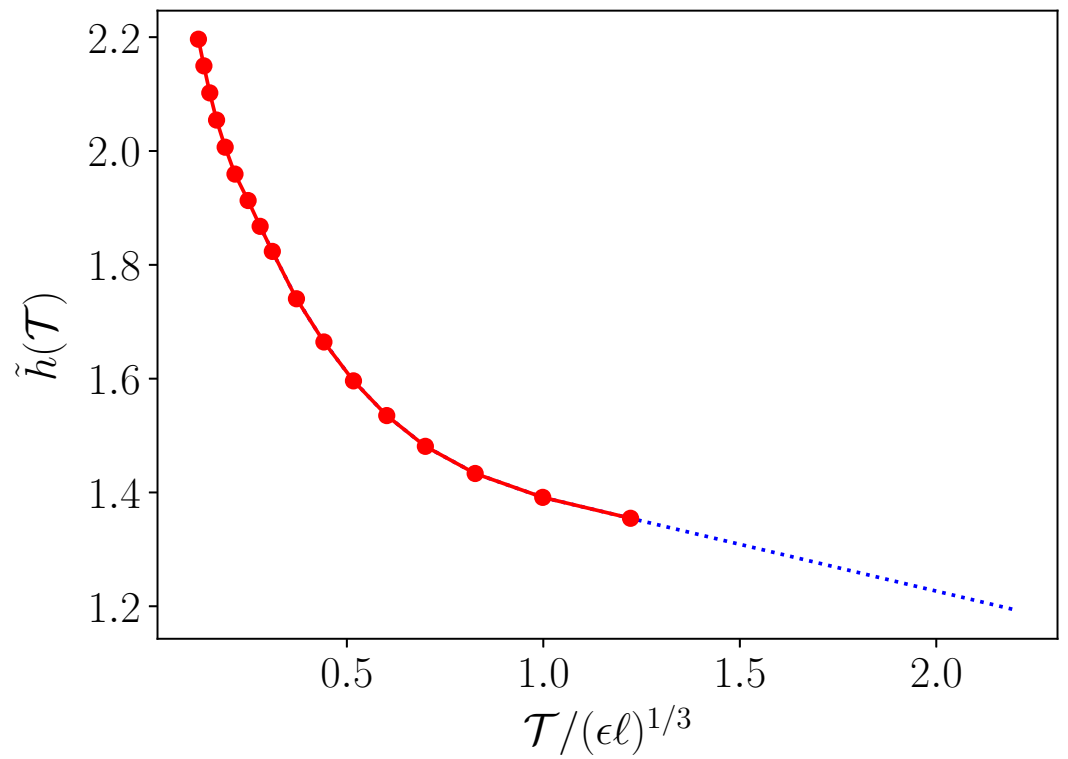

FIG. 8. Matching function linking the velocity increment to a value of local Hölder exponent at the scale $\ell_{d}=1.8 \eta$. The red curve and dots correspond to the result of the procedure described in Section II D. The dotted blue line is the extrapolation used when the velocity increment is outside the range of the red curve. 
"filament" and "sheet", depending on two invariants $Q$ and $R$. As the tools introduced in section II are based on wavelet transforms, we use a mollified version of these VGT invariants with a Mexican wavelet transform at the same (inertial or dissipative) scale for the sake of coherence. For an incompressible flow, this leads to:

$$
\begin{aligned}
Q & =-\frac{1}{2} \operatorname{Tr}\left(A_{\ell}^{2}\right), \\
R & =-\operatorname{det}\left(A_{\ell}\right)
\end{aligned}
$$

where:

$$
A_{\ell, i j}=-\int \nabla_{j} G^{\ell}(\boldsymbol{y}) u_{i}(\boldsymbol{x}+\boldsymbol{y}) \mathrm{d} \boldsymbol{y} .
$$

with $G^{\ell}$ the Mexican wavelet at scale $\ell$.

We then follow Debue [29] and compute joint Probability Density Functions (PDF) of the invariants $Q$ and $R$, as well as conditional averages of the energy transfer $D_{I}^{\ell}$ and the local Hölder exponent $\tilde{h}$ at given $Q$ and $R$. The results for the Run I, computed over 10 snapshots of velocity at an inertial scale $\ell_{0} \approx 1.4 \lambda$, are shown in figure 9 . Because of the use of wavelet transforms, our results are smoother than Debue [29]. However, the qualitative observations stay the same. We obtain the well-known droplet shape for the distribution of the invariants $Q$ and $R$ in figure 9a. For joint PDF with the local energy transfer, displayed figure $9 \mathrm{~b}$, we recover strong positive energy transfer along the Vieillefosse line in the lower right quadrant, corresponding to the limit between vortex compression and sheets, and in the vortex stretching region. Meanwhile, we observe negative energy transfers both in the vortex stretching and the vortex compressing regions for high values of $Q$.

However, the local Hölder exponents follow a different distribution. Overall, we observe lower regularity whenever the invariants $Q$ and $R$ are large in absolute value. This is true in particular but not only in the vortex region. This result is expected, as high values of the VGT invariants come with strong velocity gradients, which means a low regularity. This confirms the observation made by Nguyen et al. [19] that the structure of low local Hölder exponents are not exactly localized in the same regions as the structure of high energy transfers and are not limited to the same topologies.

The previous analysis can be reproduced in the dissipative range of Run II thanks to the computations done in the previous section. As we analyze extreme irregular events at the dissipative scale, we will use the VGT invariants in order to infer the typical topologies. For this purpose, the unconditioned PDF of the invariants $Q$ and $R$ is required as a reference. 


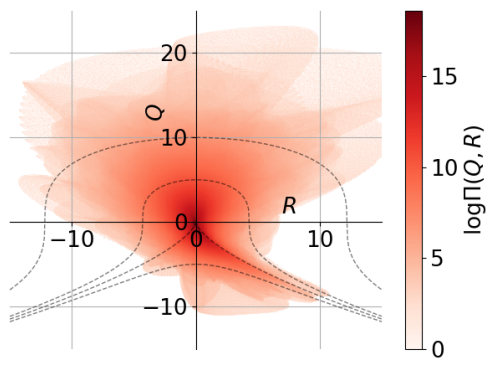

(a)

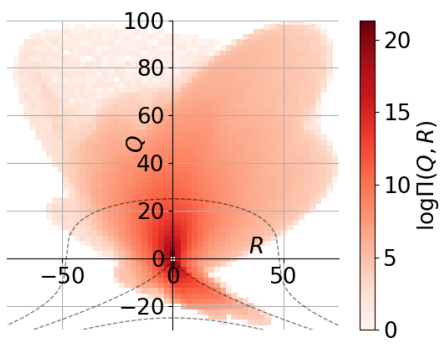

(d)

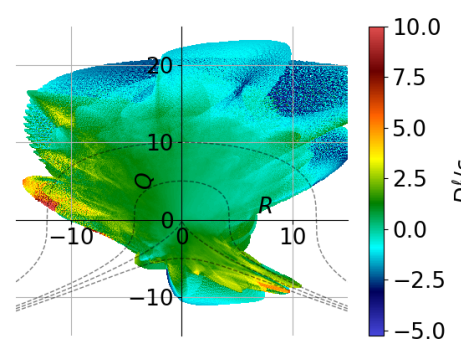

(b)

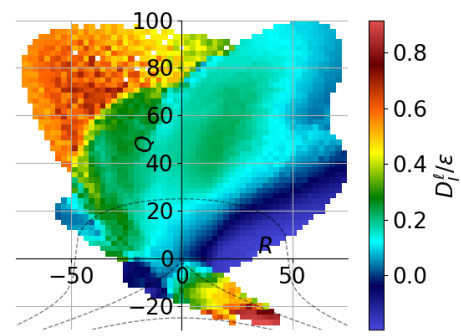

(e)

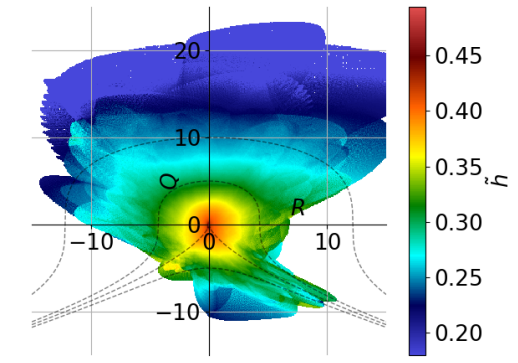

(c)

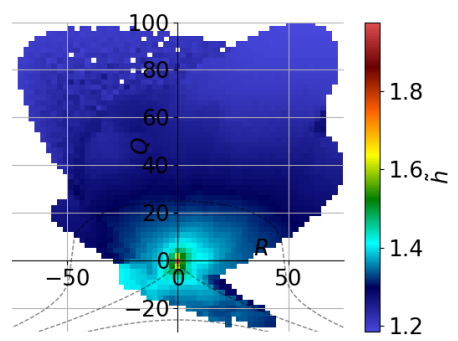

(f)

FIG. 9. (a-c): Results from Run I (inertial scale). (d-f): Results from Run II (dissipative scale). (a),(d) Joint PDF of $Q$ and $R$. (b),(e) Conditional average of the local energy transfer $D_{I}^{\ell}$ at given $Q$ and $R$. (c),(f) Conditional average of the local Hölder exponents at given $Q$ and $R$. For each figures, Vieillefosse lines (see [31]) are drawn in dashed lines. In the inertial case, the Vieillesfosse lines correspond to the equations $27 R^{2}+4 Q^{3}=-500,0,500$, and 4000. In the dissipative case, they correspond to $27 R^{2}+4 Q^{3}=-62500,0$ and 62500 .

The results, computed over 40 snapshots at the scale $\ell_{d} \approx 1.8 \eta$, are shown in figure $9 \mathrm{~d}-\mathrm{f}$. We can repeat the observations made in the inertial case: the common droplet shape is recovered for the joint PDF of $Q$ and $R$ in figure 9d. With the exception of a single excursion in the "vortex stretching" region, strong positive Duchon Robert energy transfers appear strongly concentrated in the lower right quadrant of figure 9e, corresponding to the "sheet" region. This is even more salient for Run I than for Run II. Here again this is in agreement with the observations made by Debue [29]. Finally, we observe very low values of the local Hölder exponent wherever the VGT invariants are large, similarly to the inertial case. 


\section{STATISTICAL STUDY USING UNCORRELATED DATA AT THE DISSIPA- TIVE SCALE}

\section{A. Detection of irregular events and first approach using the VGT invariants}

The first step to characterize extreme events at the dissipative scale is to get statistics using uncorrelated velocity fields. For that purpose, we use 40 velocity fields of Run II saved over 120 eddy turnover times. On those fields, we compute the local Hölder exponents using the mapping function from figure 8. We then extract all events where the local Hölder exponents get below the threshold value of $\tilde{h}=1.3$. This value is low enough that it can only be estimated using extrapolation due to the shift of $\tilde{h}$ values in the dissipative range (see section IID). It would be equivalent to finding a value of $\tilde{h} \leq 0.2$ in the inertial range. On the other hand, the threshold is high enough to detect 213 distinct events in our dataset, for a statistical analysis. We have verified that changing the value of the threshold (e.g. increasing it to $\tilde{h}=1.5$, selecting less extreme events) gives qualitatively similar results.

Before any further analysis, we can get a first grasp of the topology of the singular events using the VGT invariants. The location of the minimum of local Hölder exponent is defined as the center of the event. The VGT invariants $Q$ and $R$ computed at the center of each using the smoothed velocity gradient (see equation (10)) are shown as a scatter plot in figure 10. We first observe that, with only two exceptions, all events are either in the "vortex stretching" or the "vortex compressing" regions of the QR-plane. Moreover, there is a bias toward the former as more than $75 \%$ of the events detected verify $R<0$. This bias is stronger than what is observed in the whole domain in figure 9, which implies that the vortex stretching favors quasi singular structures. This observation is in agreement with other studies relating singularities to vortex stretching (e.g [8] in the case of Euler equations).

\section{B. Visualization of singular events}

The next step is to observe the events individually. The mean velocity over a cubic subdomain of side $1.7 \lambda$ centered on the point of minimal local Hölder exponent is subtracted in order to reduce the influence of the large scale motion in the visualizations.

For each events, we compute the local Hölder exponent, the inter scale energy transfer 


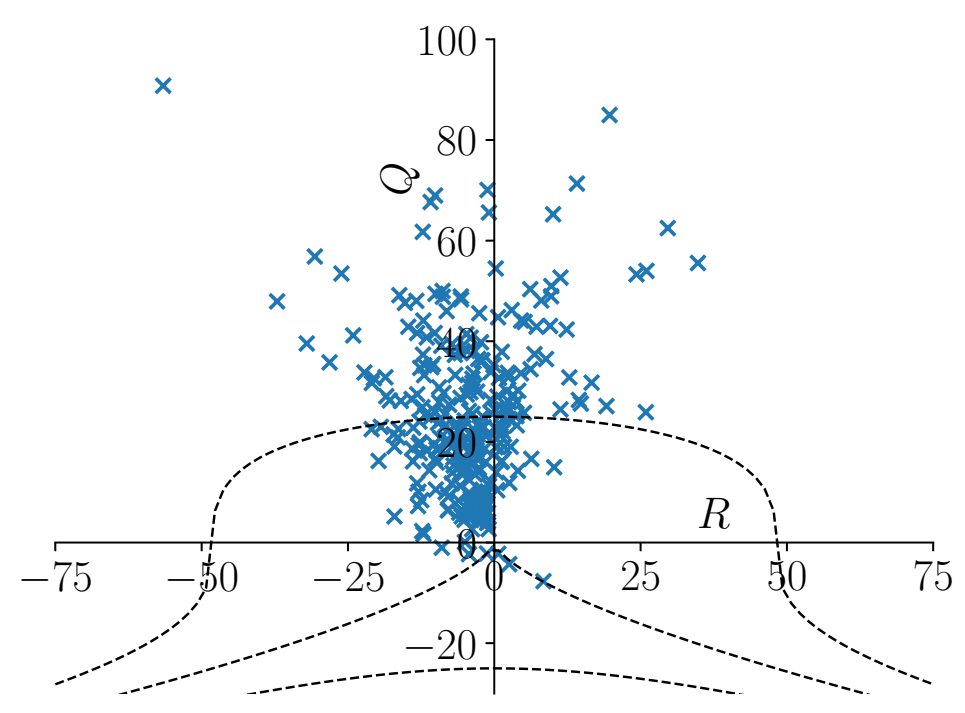

FIG. 10. Scatterplot of the mollified velocity gradient tensor invariants $Q$ and $R$ at the location of minimal local Hölder exponent for the 213 extreme events extracted at the dissipative scale. Three Vieillefossse lines at $27 R^{2}+4 Q^{3}=-62500,0$ and 62500 are drawn in dashed lines.

$D_{I}^{\ell}$ (from equation (3)), the viscous dissipation at this scale $D_{\nu}^{\ell}$ (from equation (4)), the smoothed velocity gradient tensor invariants $Q$ and $R$ (from equation (10)), as well as the vorticity.

A first overview of the events detected leads to two observations. First, we verify visually the conclusion obtained through the use of the VGT invariants: the streamlines of velocity for most events are typical of vortices. In addition, the isosurfaces of local Hölder exponents $\tilde{h}$ are seemingly oriented in the same direction as the lines of vorticity. The second observation is that the structures of low local Hölder exponent appear to be surrounded by a pair of structures of strong energy transfer $D_{I}^{\ell}$, in a way similar to what was observed in figure 6 .

For the following analysis, we reoriented all events in the same way in order to compare and classify them. The method chosen is as follow:

- The local Hölder exponent $\tilde{h}$ is the most relevant scalar for our study. In consequence, the $X$-axis was aligned with the the first eigenvector $\boldsymbol{e}_{1}$ of the covariance matrix of $\tilde{h}$.

- As irregular events appear to be vortices, we want all vortices to face the same direction. The direction of the first axis is thus chosen such that $\omega_{x}>0$ at the center. 
- From the structures of $D_{I}^{\ell}$ and $D_{\nu}^{\ell}$, it appears that irregular events are not axisymmetrical. This justifies the definition of a second axis, orthogonal to the first one. Using the first eigenvector $\boldsymbol{e}_{2}$ of the covariance matrix of $D_{\nu}^{\ell}$, the events were reoriented such that $\boldsymbol{e}_{1} \times \boldsymbol{e}_{2}$ is along the $Z$-axis.

We have not found any feature which would privilege a direction for the second axis. This means that rotating the event by an angle $\pi$ around $\boldsymbol{e}_{1}$ still fulfills the conditions. See Supplemental Materials [38] for visualizations of all events found.

Using the data collected, we can repeat the classification for the extreme events produced by Debue [29] in experimental datasets, using extreme events of $D_{I}^{\ell}$. In that work, the author distinguishes two types of vortices based on the behavior of the helicity: the roll vortices and the screw vortices. The roll vortices corresponds to events where the helicity changes sign over the structures as opposed to screw vortices which have an helicity of constant sign. Debue also mentions that the difference between the two types of events might just be a simple Galilean transformation.

The predominant structure in our dataset is the one corresponding to roll vortices. Such a structure is illustrated in figure 11a. In some cases, the change of helicity occurs slightly away from the point of minimum local Hölder exponent. We can attribute this observation to a remnant contribution of the large scale velocity. Meanwhile, screw vortices rarely appear. One could interpret the aforementioned off centered roll vortices, like for example in figure 11b, as screw vortices. However, no qualitative difference between those two types of vortices is observed in any of the observable used here. This leads to the conclusion that, as it was hypothetized by Debue [29], those two structures are the same up to a Galilean transformation.

The work of Debue [29] identifies a third type of structure called "U-turn". It is characterized by a sharp change of direction of the velocity streamlines. No "U-turns" is observed in our dataset. The closest match are tight vortices, such that they could be identified as "U-turn" if observed at a lower resolution with the addition of experimental noise. However, we cannot reject the possibility that "U-turns" fit the criterion based on local energy transfer but not the one based on local Hölder exponents, which would mean they are not detected in our case. Another possibility is that "U-turns" correspond to an earlier phase in the development of an irregular event, such that the local Hölder exponent is still too high to be detected by our method. Finally, it could also be a structure appearing only at higher 


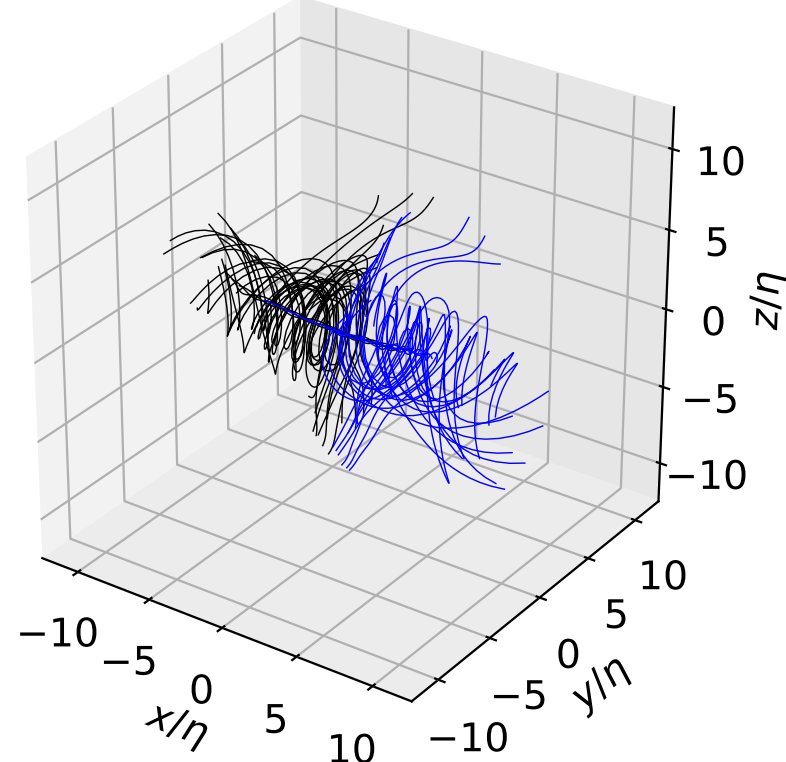

(a)

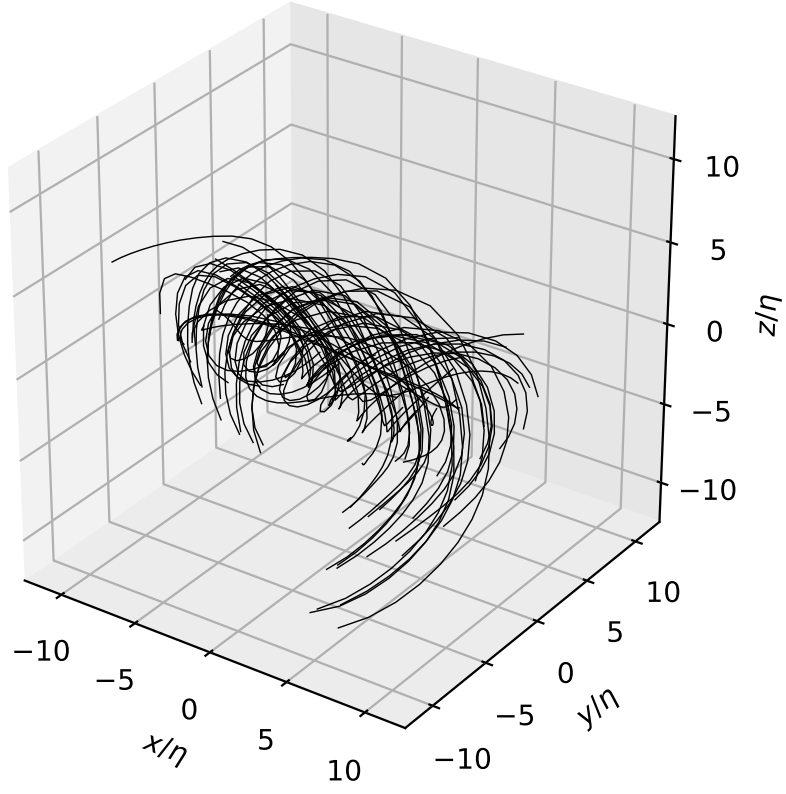

(b)

FIG. 11. (a): Streamlines of velocity for a "roll-vortex" event (event 2 from the Supplemental Materials [38]). Two different colors of streamlines are used to visualize the change of direction: the axial velocity is oriented away from the $x=0$ plane. (b): Streamlines of velocity identifiable to a "screw-vortex" event (event 107 from the Supplemental Materials [38]). The axial velocity is oriented toward $x>0$.

Reynolds number. If the last hypothesis is true, we would not be able to observe this type of event through the simulations performed in this study. This hypothesis is supported by the high Reynolds number simulation performed by Yeung [16], which exhibit a different type of event, which might correspond to "U-turns".

\section{Typical event}

As we have extracted and reoriented the extreme events along a common direction, we can average them in order to extract a "typical" event. This is justified by the fact most events share the common structure of "roll vortices". The few exceptions consist mainly in complex patterns which could be related to several events happening close to each others. Those events are kept in the average in order to avoid introducing any bias. We expect that these events, with no clear common feature, will have little contribution to the average. 


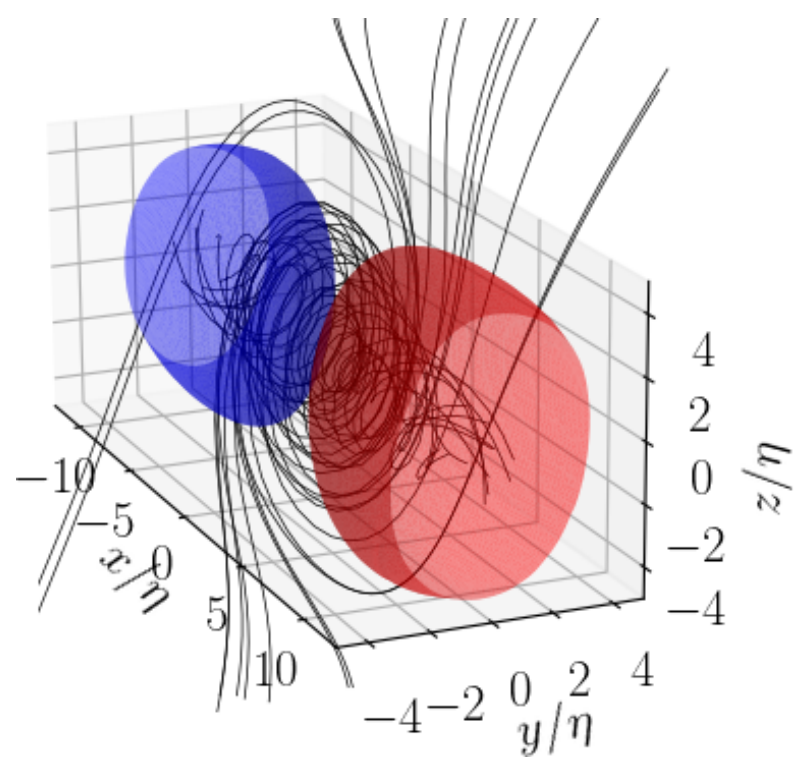

(a)

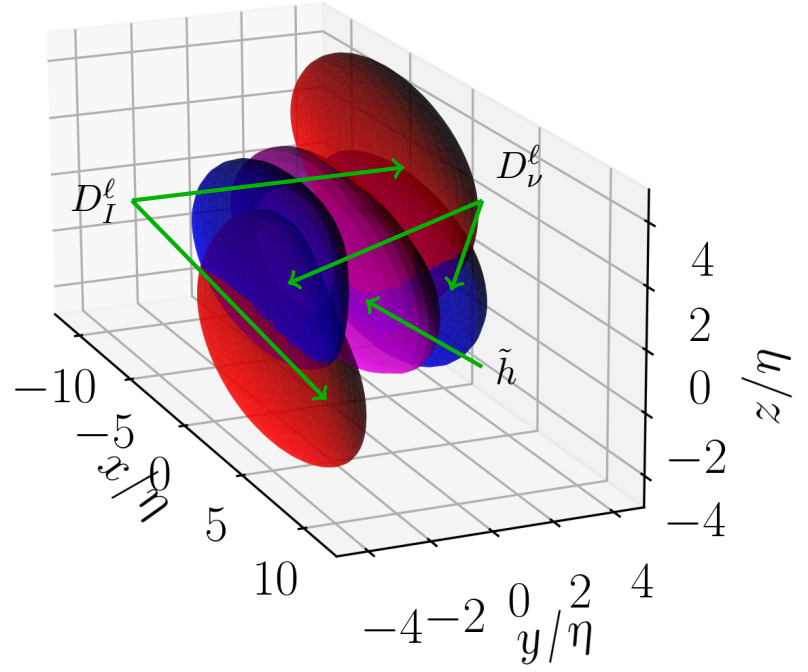

(b)

FIG. 12. (a): Streamlines of velocity. The axial velocity is oriented away from the $x=0$ plane. The red (resp. blue) isosurface corresponds to an helicity of $H=2.5$ (resp. $H=-2.5$ ). (b): The magenta isosurface corresponds to the local Hölder exponent $\tilde{h}=1.35$. The red isosurface corresponds to $D_{I}^{\ell}=2$.pdfilon. The blue isosurface corresponds to $D_{\nu}^{\ell}=4 \epsilon$.

The average is taken over the 213 most singular events extracted, corresponding to all events with a local Hölder exponent $\tilde{h}<1.3$ at the scale $\ell=1.8 \eta$. As before, we verified that changing the value of the threshold gives qualitatively similar ersults. In this case, choosing a lower threshold leads to more extreme values of averaged local Hölder exponent, energy transfers and vorticity, but preserves the topology of the averaged event. We provide a visualization for this averaged event in figure 12 as well as in the Supplemental Materials [38] for more details. Note that the scalars displayed are the averaged values of the exponent $\tilde{h}$, the energy transfer $D_{I}^{\ell}$ and the energy dissipation $D_{\nu}^{\ell}$. Because those quantities are not linear functions of the velocity field, they do not correspond to the values that would be computed from the averaged velocity field, materialized by the streamlines.

The streamlines of the "typical" event match the pattern of roll vortices, with a change of helicity at the $x=0$ plane, corresponding to the location of the minimum of local Hölder exponent. This seems to be the typical behavior for extremely singular events. In particular, one might recognize in the streamlines the profile of a Burgers vortex [32]. An axisymmetrical Burgers vortex can be characterized by its vorticity profile: 


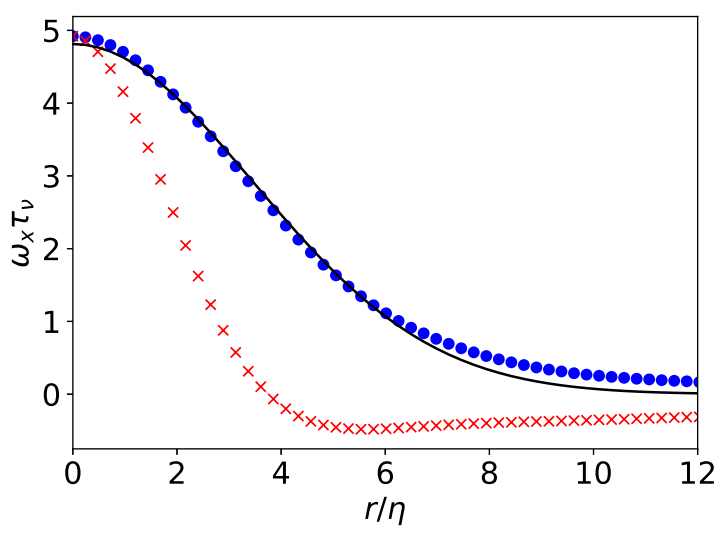

(a)

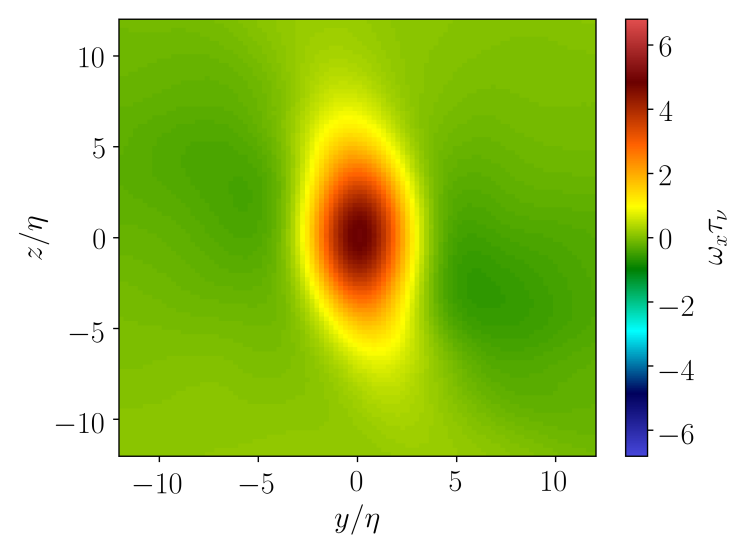

(b)

FIG. 13. (a) Fit of the component of the vorticity $\omega_{x}$ in the $x=0$ plane for the averaged event. The vorticity along the $z$ (resp. $y$ ) direction is represented with blue circles (resp. red crosses) while the Burgers fit is shown as a black continuous curve. (b) Colormap of the component of the vorticity $\omega_{x}$ in the $x=0$ plane for the averaged event.

$$
\omega_{x}(r)=\frac{\Gamma}{2 \pi \nu} \exp \left(-\frac{\sigma r^{2}}{2 \nu \eta^{2}}\right) .
$$

We can fit the profile of vorticity in the plane $x=0$, which gives the values $\Gamma \tau_{\nu} / 2 \pi \nu=5$ and $\sigma / \nu=0.08$. The fit works very well in the $z$ direction but fails in the $y$ direction, as illustrated in figure 13a. Note that the non axisymmetric Burger vortex would not provide a better result as the vorticity changes sign in the $y$ direction. This asymmetry in the vorticity can be visualized in figure $13 \mathrm{~b}$.

We likewise observe a very strong non axisymmetry of the averaged $D_{I}^{\ell}$ and $D_{\nu}^{\ell}$. While the invariance by a rotation of $\pi$ around the $x$-axis is a consequence of the orientation of the events before averaging, we do confirm the presence of structures of $D_{I}^{\ell}$ and $D_{\nu}^{\ell}$ on both sides of the axis in individual events. A possible explanation to these non axisymmetric structures would be that extreme events correspond to an interaction between vortices of different strength, so that the weakest, least singular vortex is canceled out in the process of averaging. Furthermore, the reversal of helicity appears similar to what happens around a vortex reconnection [3]. This is also reminiscent of the classical works from Couder [e.g 33, 34] analyzing the formation and death of vortex filaments in turbulence, even if the structures observed here are at a smaller scale than those of the cited papers. In the sequel, 
we refine such hypothesis using time resolved data. Indeed, because of the way those events were extracted, it is not clear whether the snapshots are captured prior, during or subsequent to the time for which the event is the most singular.

\section{Link with the energy transfers}

While there are extreme events with negative energy transfer $D_{I}^{\ell}$, i.e. energy going to the larger scales, the averaged event only transfers energy to smaller scales so that the average of the term $D_{I}^{\ell}$ stays positive around the region of low regularity.

We further observe that the energy transfer $D_{I}^{\ell}$, and the dissipation energy transfer $D_{\nu}^{\ell}$, do not reach their maxima at the same location. Most of the energy transfer happens slightly upstream on the streamlines from the peak of dissipation. We currently do not have a physical explanation for this phenomenon.

\section{E. Temporal evolution of an event.}

We use the time resolved part of our dataset to try and uncover the generation mechanism for extreme events. During the time period covered, 9 events for which the minimum of local Hölder exponent is kept under the threshold of $\tilde{h}=1.3$ for at least 10 snapshots (or at least 1.5 eddy turnover times $t_{\eta}$ ) are found. The choice of this thresholds k.pdf the number of events to analyze low, as the analysis of time series is more time consuming than the one of isolated snapshots. Movies of the corresponding time evolution can be found in the Supplemental Materials [38]. Each events is characterized by isosurfaces of vorticity (with red and blue coloring corresponding to positive and negative helicity respectively), local Hölder increments, energy transfers $D_{I}^{\ell}$ and dissipation $D_{\nu}^{\ell}$. We also plot the time evolution of the extrema for these quantities.

Those results appear to confirm the relation between extreme events of local Hölder exponent and vortex interaction. Indeed, the events observed are localized around two or more vortices, with possible vortex reconnections. One of these events is represented in figures 14 and 15. This event has been selected for its strength and simplicity, making it easier to visualize. For this event, the structures of high energy transfer and energy dissipation and low local Hölder exponents start to appears in figure 14d, before the onset 
of the interaction between two vortices. We can identify the structure of the typical event previously discussed in figure 14e, when interaction with another vortex coming from $z>0$ starts to deform the vortex in the figure 14c. When the vortices come close in figure 14f, the event deviates from the typical structure. In this particular case, the structures of strong energy dissipation $D_{\nu}^{\ell}$ move away from the structures of low local Hölder exponents $\tilde{h}$ and high energy transfer $D_{I}^{\ell}$ during the second part of the interaction visualized in figure 15 . The structures shown in figure 15 differ from the typical event from figure 12 . This could explain the complex structures observed in the uncorrelated dataset. It should be noticed that the local Hölder exponents becomes greater than the threshold of 1.3 before the end of the interaction.

A timeline of this particular event is provided in figure 16. It shows the evolution of the maxima for the different scalars used during the event. Note that the event chosen is particularly singular, as the local Hölder exponent drops to almost $h=1.2$. Such value is very low, as can be interpreted from the extrapolation needed in figure 8 to reach it.

From the time evolution, the most singular instant happens during the interaction, at the time corresponding to figure 14c. At this time, the local Hölder exponents reach their minimum and the vorticity and the energy dissipation reach their maximum. We observe that the maximum of energy transfer $D_{I}^{\ell}$ is reached slightly earlier. Such observation can be repeated for other events in the dataset. This matches with the previous observation that regions of high energy transfer are located upstream on the streamlines of velocity. This observation could hint toward the formation mechanism of quasi singularities.

\section{DISCUSSION}

In this paper, we have used the tool developed by Nguyen et al. [19] to extract extremely singular events from numerical turbulent velocity fields. These events have been analyzed using local energy transfers at the Kolmogorov scale as well as the velocity gradient tensor invariants. From these analysis, we derive several characteristics common to the most singular events.

The first observation is that most events are "roll vortices", i.e. vortices with a change of helicity at the most singular point. The average structure computed from the most singular events is similar to a Burgers vortex, with a strong non axisymmetry. In this work, we do 


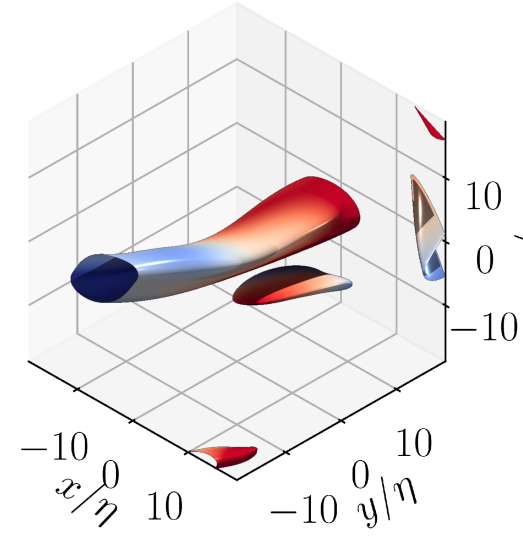

(a)

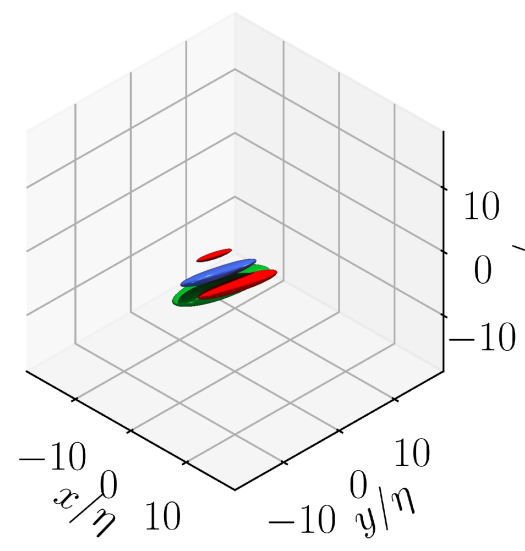

(d)

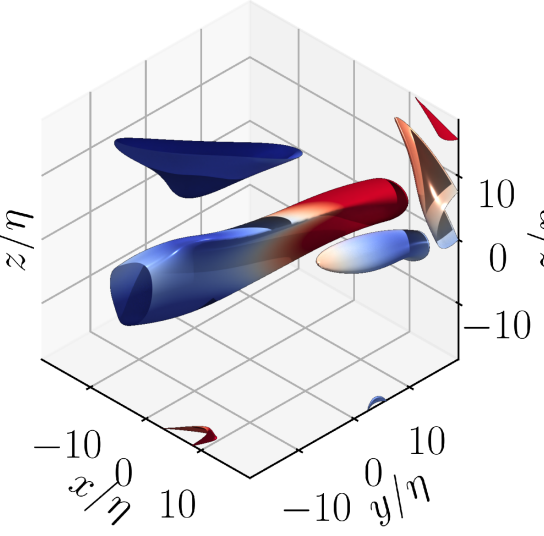

(b)

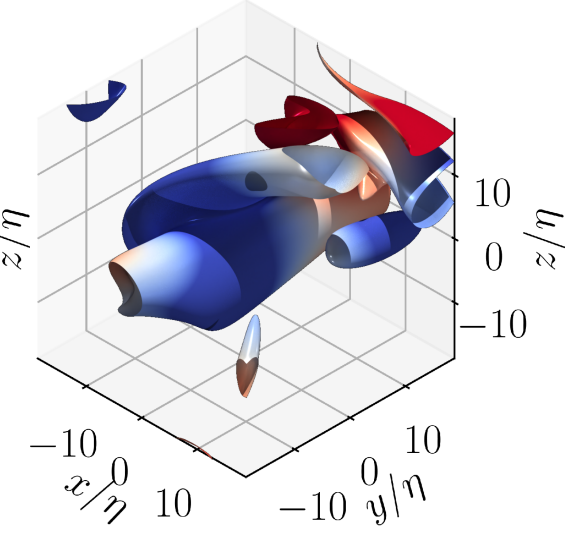

(c)

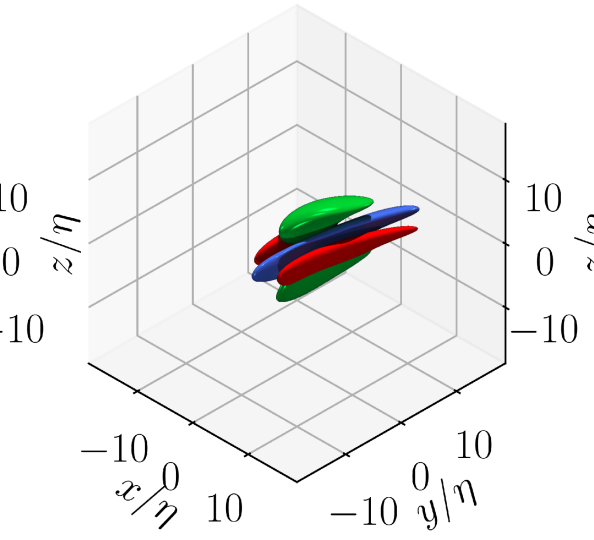

(e)

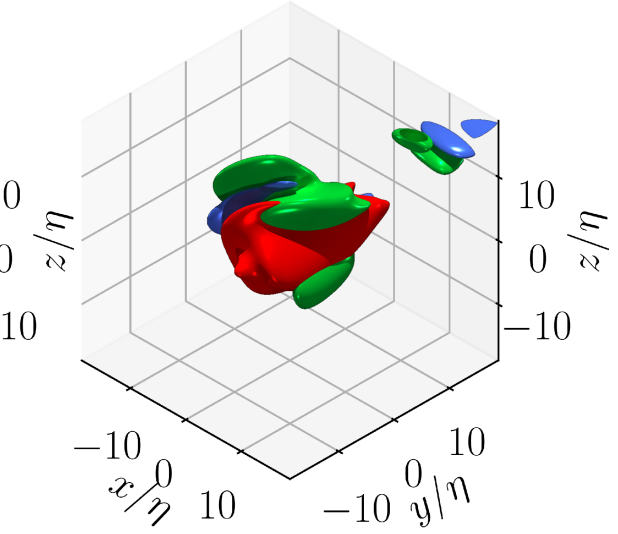

(f)

FIG. 14. Snapshots of time resolved event: (a-d): Before the interaction. (b-e): Beginning of interaction. (c-f) Time of the minimum of $\tilde{h}$. (a-b-c): Isosurfaces of vorticity $\omega t_{\eta}=23$ colored in red and blue respectively for positive and negative helicity. (d-e-f) Isosurface $\tilde{h}=1.3$ in blue, $D_{I}^{\ell} / . p d$ filon $=4$ in red, $D_{\nu}^{\ell} / \epsilon=6$ in green.

not recover the "U-turns" observed by Debue [29] and possibly present in the simulation by Yeung [16], both of these studies having been realized at a higher Reynolds number. Note however that trajectories around a Burgers vortex typically produce both "roll vortices" and "U-turns", as recently discussed by Moffat [35].

The second observation is that the local energy transfers around singular events are both away from the vortex axis and strongly non axisymmetric. The averaged event, as well as the individual events, exhibit two regions of energy transfer to lower scales $D_{I}^{\ell}$ located on both side of the main axis. The same observation can be done about the viscous energy dissipation $D_{\nu}^{\ell}$. To explain these facts, we emit the hypothesis that the extreme events are caused by 


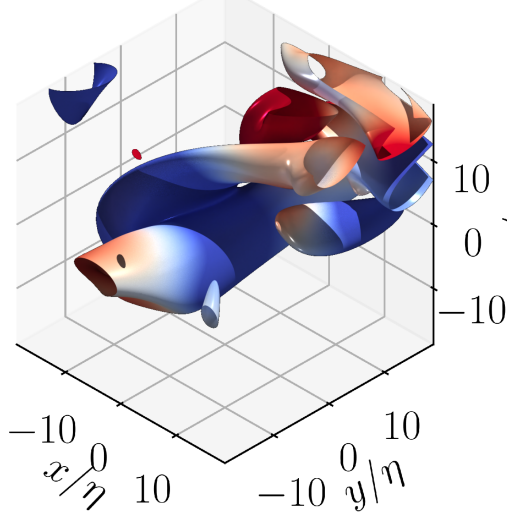

(a)

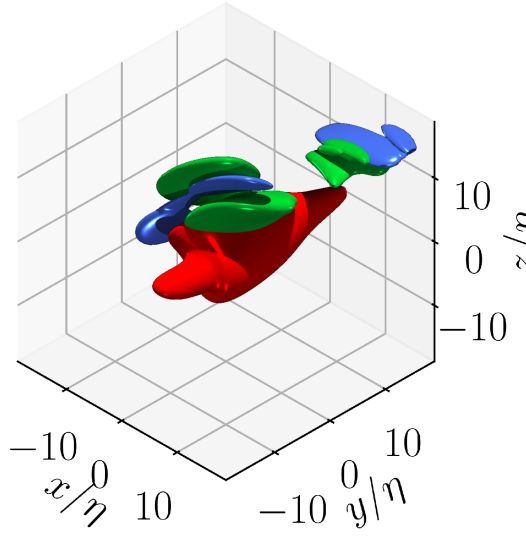

(c)

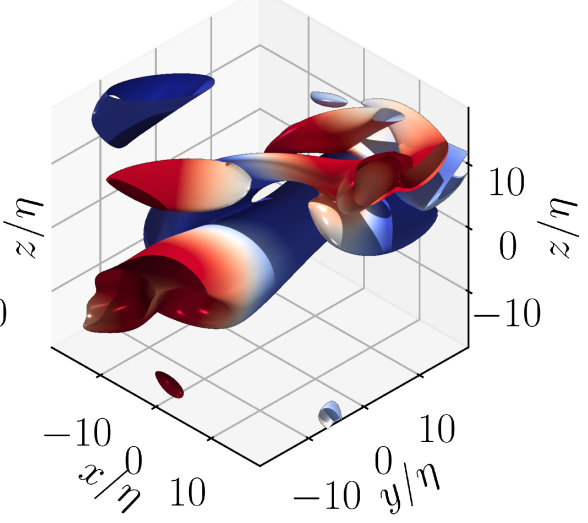

(b)

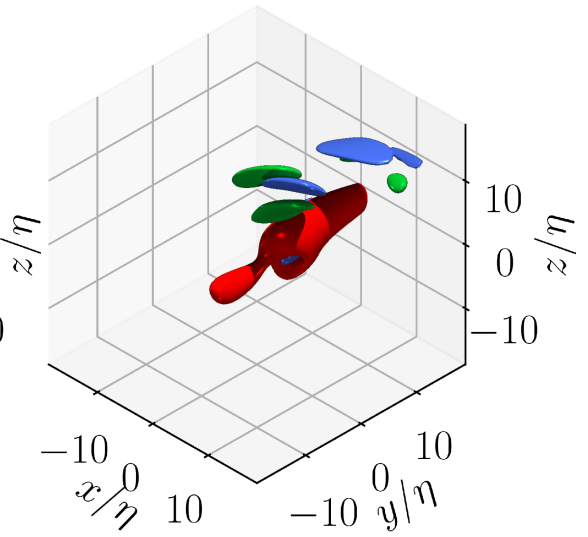

(d)

FIG. 15. Snapshots of time resolved event: (a-c): Toward the end of interaction. (b-d): Slightly before the local Hölder exponent $\tilde{h}$ grows back above 1.3. (a-b): Isosurfaces of vorticity $\omega t_{\eta}=23$ colored in red and blue respectively for positive and negative helicity. (c-d) Isosurface $\tilde{h}=1.3$ in blue, $D_{I}^{\ell} / . p d f i l o n=4$ in red, $D_{\nu}^{\ell} / \epsilon=6$ in green.

interactions between at least two vortices, which would explain the non axisymmetry. This hypothesis is supported by the analysis of the time resolved data which shows that the singular events are associated with several vortices close to one another. Some cases involve a vortex reconnection, but we are unable to confirm the impact on the singularity of the event.

As those results are obtained from simulation data, all events detected are regularized at lower scale by the numerical scheme. In this case, the pseudo spectral method is filtering out wave numbers higher than $k_{\max }$. As the small scales are expected to have a non negligible contribution for very singular events, using simulation data might have prevented us from 


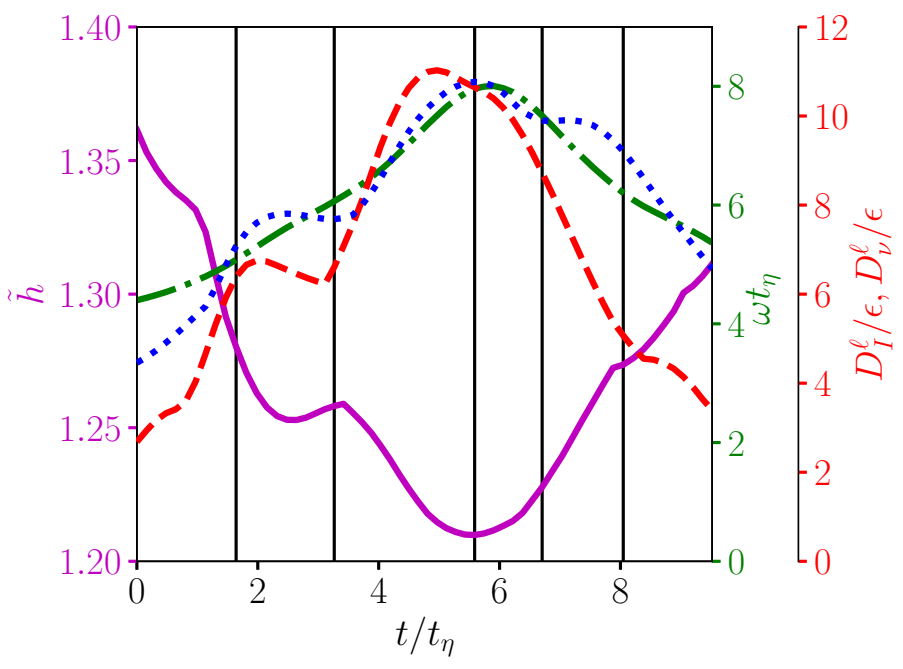

FIG. 16. Time evolution of the local Hölder exponents (in magenta, continuous line, left axis), the vorticity $\omega t_{\nu}$ (in green, dot-dashed line, middle axis), the local energy transfer $D_{I}^{\ell} / . p d f i l o n$ (in red, dashed line, rightmost axis) and the energy dissipation $D_{\nu}^{\ell} / \epsilon$ (in blue, dotted line, rightmost axis). The vertical lines indicates the times of the five snapshots represented in figures 14 and 15 .

observing more singular events. It would be interesting to reproduce this study using very well resolved experimental data, in order to validate the conclusions of the present work.

Another limit to this work is the relatively modest microscale Reynolds number of the simulations used. This limitation is imposed by the high resolution required for the study of potentially singular events and the relatively high computational cost of the post-processing limiting the size of the fields. It would nevertheless be interesting to apply the current method to much larger simulation data (e.g. the previously cited work by Yeung [16]).

Possible extensions to this work could involve the study of different types of flows, including turbulence induced by Richtmyer-Meshov or Rayleigh-Taylor instabilities [36, 37]. Such extension would require to apply the method presented in this paper to inhomogeneous and/or anisotropic flows. While the present method makes no particular hypothesis on the isotropy of the flow, we can expect difficulties when processing strong inhomogeneities. Indeed, this method requires the evaluation of multiple statistical averages, with a high requirement on the size of the statistical set related to the rarity of the events we want to detect. Strong statistical inhomogeneities would prevent us from taking statistics over space, and thus reduce the size of the usable statistics in each snapshot of velocity fields. 
This would in turn greatly increase the number of snapshots required to estimate local values of Hölder exponent.

FN has been funded by École Normale Supérieure and was granted access to the HPC resources of IDRIS under the allocation A0062A01741 made by GENCI (Grand Équipement National de Calcul Intensif). This work was funded by the ANR EXPLOIT, grant agreement no. ANR-16-CE06-0006-01.

[1] G. K. Batchelor and A. A. Townsend. The nature of turbulent motion at large wave-numbers. Proceedings of the Royal Society of London. Series A. Mathematical and Physical Sciences, 199(1057):238-255, 1949.

[2] A. Vincent and M. Meneguzzi. The dynamics of vorticity tubes in homogeneous turbulence. Journal of Fluid Mechanics, 258:245-254, 1994.

[3] J. Yao and F. Hussain. A physical model of turbulence cascade via vortex reconnection sequence and avalanche. Journal of Fluid Mechanics, 883:A51, 2020.

[4] Y. Kimura and H.K. Moffatt. Reconnection of skewed vortices. Journal of Fluid Mechanics, 751:329-345, 2014.

[5] U. Frisch. Turbulence: the legacy of AN Kolmogorov. Cambridge University Press, 1995.

[6] J. Leray. Sur le mouvement d'un liquide visqueux emplissant l'espace. Acta Mathematica, 63(1):193-248, 1934.

[7] A. Pumir and E.D. Siggia. Development of singular solutions to the axisymmetric Euler equations. Physics of Fluids A: Fluid Dynamics, 4(7):1472-1491, 1992.

[8] R.M. Kerr. Evidence for a singularity of the three-dimensional, incompressible Euler equations. Physics of Fluids A: Fluid Dynamics, 5(7):1725-1746, 1993.

[9] R. Grauer and T. C. Sideris. Finite time singularities in ideal fluids with swirl. Physica D: Nonlinear Phenomena, 88(2):116-132, 1995.

[10] C. Uhlig and J. Eggers. Singularities in cascade models of the Euler equation. Zeitschrift für Physik B Condensed Matter, 103(1):69-78, 1997.

[11] G. Luo and T. Y. Hou. Potentially singular solutions of the 3D axisymmetric Euler equations. Proceedings of the National Academy of Sciences, 111(36):12968-12973, 2014.

[12] R.M. Kerr. The role of singularities in Euler. In Small-Scale Structures in Three-Dimensional 
Hydrodynamic and Magnetohydrodynamic Turbulence, pages 17-23. Springer, 1995.

[13] R.M. Kerr. Vortex collapse and turbulence. Fluid Dynamics Research, 36(4-6):249, 2005.

[14] T. Grafke, H. Homann, J. Dreher, and R. Grauer. Numerical simulations of possible finite time singularities in the incompressible Euler equations: comparison of numerical methods. Physica D: Nonlinear Phenomena, 237(14-17):1932-1936, 2008.

[15] J.C. Vassilicos and J.G. Brasseur. Self-similar spiral flow structure in low Reynolds number isotropic and decaying turbulence. Physical Review E, 54(1):467, 1996.

[16] P.K. Yeung, X.M. Zhai, and R. Sreenivasan. Extreme events in computational turbulence. Proceedings of the National Academy of Sciences, 112(41):12633-12638, 2015.

[17] D. Buaria, A. Pumir, E. Bodenschatz, and P.K. Yeung. Extreme velocity gradients in turbulent flows. New Journal of Physics, 21(4):043004, 2019.

[18] D. Buaria, A. Pumir, and E. Bodenschatz. Self-attenuation of extreme events in Navier-Stokes turbulence. arXiv preprint arXiv:2009.08370, 2020.

[19] F. Nguyen, J.-P. Laval, P. Kestener, A. Cheskidov, R. Shvydkoy, and B. Dubrulle. Local estimates of Hölder exponents in turbulent vector fields. Physical Review E, 99(5):053114, 2019 .

[20] D. Kuzzay, E.-W. Saw, F. Martins, D. Faranda, J.-M. Foucaut, F. Daviaud, and B. Dubrulle. New method for detecting singularities in experimental incompressible flows. Nonlinearity, 30(6):2381, 2017.

[21] B. Dubrulle. Beyond Kolmogorov cascades. Journal of Fluid Mechanics, 867:P1, 2019.

[22] J. Duchon and R. Robert. Dissipation d'énergie pour des solutions faibles des équations d'Euler et Navier-Stokes incompressibles. Séminaire Équations aux dérivées partielles, 2000:1-10, 2000.

[23] Y. Zhou. Interacting scales and energy transfer in isotropic turbulence. Physics of Fluids A: Fluid Dynamics, 5(10):2511-2524, 1993.

[24] A.N. Kolmogorov. The local structure of turbulence in incompressible viscous fluid for very large Reynolds numbers. Dokl. Akad. Nauk SSSR, 30:9-13, 1941.

[25] P. Kestener and A. Arneodo. Generalizing the wavelet-based multifractal formalism to random vector fields: application to three-dimensional turbulence velocity and vorticity data. Physical Review Letters, 93(4):044501, 2004.

[26] G. Parisi and U. Frisch. On the singularity structure of fully developed turbulence. Turbulence 
and Predictability in Geophysical Fluid Dynamics and Climate Dynamics, 1985.

[27] R. Benzi, S. Ciliberto, R. Tripiccione, C. Baudet, F. Massaioli, and S. Succi. Extended selfsimilarity in turbulent flows. Physical Review E, 48(1):R29, 1993.

[28] A. Arneodo, N. Decoster, and S.G. Roux. A wavelet-based method for multifractal image analysis. I. Methodology and test applications on isotropic and anisotropic random rough surfaces. The European Physical Journal B-Condensed Matter and Complex Systems, 15(3):567-600, 2000 .

[29] P. Debue. Experimental approach to the problem of the Navier-Stokes singularities. PhD thesis, Université Paris-Saclay, 2019.

[30] M. S. Chong, A. E. Perry, and B. J. Cantwell. A general classification of three-dimensional flow fields. Physics of Fluids A: Fluid Dynamics, 2(5):765-777, 1990.

[31] P. Vieillefosse. Internal motion of a small element of fluid in an inviscid flow. Physica A: Statistical Mechanics and its Applications, 125(1):150-162, 1984.

[32] J. M. Burgers. A mathematical model illustrating the theory of turbulence. In Advances in applied mechanics, volume 1, pages 171-199. Elsevier, 1948.

[33] O. Cadot, S. Douady, and Y. Couder. Characterization of the low-pressure filaments in a three-dimensional turbulent shear flow. Physics of Fluids, 7(3):630-646, 1995.

[34] S. Douady, Y. Couder, and M.E. Brachet. Direct observation of the intermittency of intense vorticity filaments in turbulence. Physical Review Letters, 67(8):983, 1991.

[35] H.K. Moffatt. Extreme events in turbulent flow. submitted, 2020.

[36] Y. Zhou. Rayleigh-taylor and Richtmyer-Meshkov instability induced flow, turbulence, and mixing. ii. Physics Reports, 723-725:1-160, 2017.

[37] Y. Zhou. Rayleigh-taylor and Richtmyer-Meshkov instability induced flow, turbulence, and mixing. i. Physics Reports, 720-722:1-136, 2017.

[38] See Supplemental Materials at [URL will be inserted by publisher] for snapshots and movies of the extreme events. 